\title{
ARTICLE
}

Cellular and Molecular Biology

\section{A novel TRPM7/O-GlcNAc axis mediates tumour cell motility and metastasis by stabilising c-Myc and caveolin-1 in lung carcinoma}

Sudjit Luanpitpong $\mathbb{D}^{1}$, Napachai Rodboon ${ }^{1}$, Parinya Samart ${ }^{2}$, Chanida Vinayanuwattikun ${ }^{3}$, Siwaporn Klamkhlai ${ }^{4}$, Pithi Chanvorachote ${ }^{5}$, Yon Rojanasakul ${ }^{6}$ and Surapol Issaragrisil ${ }^{1,7,8}$

BACKGROUND: Calcium is an essential signal transduction element that has been associated with aggressive behaviours in several cancers. Cell motility is a prerequisite for metastasis, the major cause of lung cancer death, yet its association with calcium signalling and underlying regulatory axis remains an unexplored area.

METHODS: Bioinformatics database analyses were employed to assess correlations between calcium influx channels and clinical outcomes in non-small cell lung cancer (NSCLC). Functional and regulatory roles of influx channels in cell migration and invasion were conducted and experimental lung metastasis was examined using in vivo live imaging.

RESULTS: High expression of TRPM7 channel correlates well with the low survival rate of patients and high metastatic potential. Inhibition of TRPM7 suppresses cell motility in various NSCLC cell lines and patient-derived primary cells and attenuates experimental lung metastases. Mechanistically, TRPM7 acts upstream of $O$-GlcNAcylation, a post-translational modification and a crucial sensor for metabolic changes. We reveal for the first time that caveolin-1 and c-Myc are favourable molecular targets of TRPM7/O-GICNAc that regulates NSCLC motility. O-GIcNAcylation of caveolin-1 and c-Myc promotes protein stability by interfering with their ubiquitination and proteasomal degradation.

CONCLUSIONS: TRPM7/O-GICNAC axis represents a potential novel target for lung cancer therapy that may overcome metastasis.

British Journal of Cancer (2020) 123:1289-1301; https://doi.org/10.1038/s41416-020-0991-7

\section{BACKGROUND}

Calcium is a ubiquitous secondary messenger that initiates various signal transduction cascades and influences numerous signalling pathways, controlling fundamental cellular processes, such as cell proliferation, differentiation and cell death. ${ }^{1-3}$ Since these processes are relevant to tumorigenesis, alterations of calcium signalling and homeostasis are thought, if not have been shown, to be implicated in tumorigenesis and tumour progression. Changes in cytosolic free calcium $\left(\mathrm{Ca}^{2+}\right)$ concentrations in response to various extracellular stimuli are typically fuelled by a combination of calcium influx through specialised $\mathrm{Ca}^{2+}$-permeable channels and release of $\mathrm{Ca}^{2+}$ from intracellular stores-usually endoplasmic reticulum (ER). Intracellular calcium signalling has been observed to be a complex system containing a myriad of proteins, also known as $\mathrm{Ca}^{2+}$ toolkit, that are able to bind to $\mathrm{Ca}^{2+}$ for their coupling to downstream signalling pathways and finally switching it off by removing the $\mathrm{Ca}^{2+}$ from the cytosol. ${ }^{1}$ In the context of cancers, major rearrangement of $\mathrm{Ca}^{2+}$ channels by means of changes in their expression and/or function when compared to normal cells has been reported, but there is very limited data concerning these channels and cancer cell motility, particularly in lung cancer. ${ }^{4-6}$

Lung cancer is the leading cause of cancer death that kills more than one million people worldwide each year, due largely to diagnosis at late stages with local or distant organ metastasis. ${ }^{7}$ Cancer cell migration and invasion into surrounding tissues through basement membranes are an initial step in metastasis, allowing the dissemination of primary tumour cells into blood circulation to establish new or secondary tumour sites. ${ }^{8,9}$ Recent studies have emphasised that $\mathrm{Ca}^{2+}$ channels that are associated with aggressive cancer behaviours are primarily non-voltageactivated channels and belong to transient receptor potential (TRP) superfamily and Orai family. ${ }^{10-12}$ The TRP proteins that are frequently modified during cancer progression include TRP canonical (TRPC1 and 6), TRP vanilloid (TRPV2 and 6) and TRP melastatin (TRPM7 and 8). Among those, TRPM7 has earlier been

\footnotetext{
${ }^{1}$ Siriraj Center of Excellence for Stem Cell Research, Faculty of Medicine Siriraj Hospital, Mahidol University, Bangkok, Thailand; ${ }^{2}$ Department of Immunology, Faculty of Medicine Siriraj Hospital, Mahidol University, Bangkok, Thailand; ${ }^{3}$ Department of Medicine, Division of Medical Oncology, Faculty of Medicine, Chulalongkorn University and The King Chulalongkorn Memorial Hospital, Bangkok, Thailand; ${ }^{4}$ Department of Pathology, Faculty of Medicine Siriraj Hospital, Mahidol University, Bangkok, Thailand; ${ }^{5}$ Department of Pharmacology and Physiology, Faculty of Pharmaceutical Sciences, Chulalongkorn University, Bangkok, Thailand; ${ }^{6}$ WVU Cancer Institute and Department of Pharmaceutical

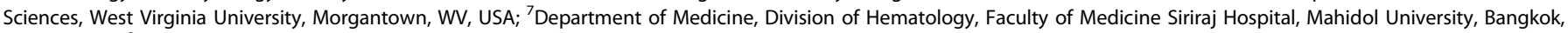
Thailand and ${ }^{8}$ Bangkok Hematology Center, Wattanosoth Hospital, BDMS Center of Excellence for Cancer, Bangkok, Thailand

Correspondence: Sudjit Luanpitpong (suidjit@gmail.com)

These authors contributed equally: Napachai Rodboon, Parinya Samart
}

Received: 4 December 2019 Revised: 1 June 2020 Accepted: 1 July 2020

Published online: 20 July 2020 
shown to be aberrantly expressed in lung adenocarcinoma and squamous cell lung carcinoma and linked to cancer stem cell subpopulation and its carcinogenic potential. ${ }^{13}$ Orai1, through its interaction with $\mathrm{ER} \mathrm{Ca}^{2+}$ sensor protein STIM1 following depletion of internal $\mathrm{Ca}^{2+}$ store, activated store-operated calcium entry at the plasma membrane. ${ }^{10,14}$ Using bioinformatics database, we examined the association between TRPM7, ORAI1 and STIM1 messenger RNA (mRNA) expression and clinical prognosis in nonsmall cell lung cancer (NSCLC). We found that high expression of TRPM7 and ORAI1 correlate well with low survival rates of NSCLC patients, while patients with high expression of STIM1 exhibit a similar survival to those with lower expression. Gene repression by CRISPR/Cas9 system revealed an essential role of TRPM7, and to a lesser extent Orai1 and STIM1, in cell motility, leading us to the further investigation of TRPM7 functions, signalling cascades and downstream targets.

Metabolic reprogramming is an emerging hallmark of cancers that occurs during both malignant transformation and tumour development. ${ }^{15,16}$ O-GlcNAcylation is an essential post-translational modification (PTM) that uses the final product of nutrient flux UDPGlcNAc through the hexosamine biosynthetic pathway. As this pathway integrates amino acids, carbohydrates, fatty acids, nucleotides and energy metabolism, the extent of O-GlcNAcylation generally reflect the global metabolic dynamics in the cells. ${ }^{17,18}$ Herein, we found that $O$-GlcNAcylation plays a key regulatory role in coordinating calcium signalling via TRPM7 channel, which induces cell migration and invasion in vitro and experimental lung metastasis in vivo. We further unveiled c-Myc and caveolin-1 (Cav-1) as favourable protein-specific O-GlcNAcylation that contribute to the motility changes. Our findings suggest a crosstalk between calcium signalling and dysregulated metabolism in cancer cells that could be important in understanding lung cancer progression and metastasis, and may have clinical utility for targeted therapy of lung and other cancers whose aetiology are dependent on abnormal $\mathrm{Ca}^{2+}$ influx and O-GlcNAcylation.

\section{METHODS}

Survival analysis

Kaplan-Meier survival plots according to the expression of TRPM7, ORAI1 and STIM1 assessed by gene chip microarrays were generated using the Kaplan-Meier plotter (http://kmplot.com/ analysis/) containing clinical data of 2437 lung cancer patients. Only the data from JetSet best probe, which selects the optimal probe set for each gene, was included in the analysis. ${ }^{19,20}$

\section{Differential gene expression analysis}

mRNA expression of TRPM7 from 109 lung carcinoma tissues were from Bittner's dataset in Oncomine ${ }^{T M}$ bioinformatics database (https://www.oncomine.org/resource/login.html), which were analysed on Affymetrix U133 Plus 2.0 microarrays and grouped according to their TNM staging into N0, N1 and N2, due to the availability of data. MGEA5 expression in lung adenocarcinoma and normal tissues were from Su's dataset, which were analysed on Human Genome U133A Array, as previously described. ${ }^{21}$

\section{Cell culture and patient-derived primary cancer cells}

National Cancer Institute ( $\mathrm{NCl}$ ) lung cancer cell lines, $\mathrm{NCl}-\mathrm{H} 292$, $\mathrm{NCl}-\mathrm{H} 460, \mathrm{~A} 549$ and $\mathrm{NCl}-\mathrm{H} 23$ cells, were obtained from American Type Culture Collection (ATCC, Manassas, VA) and cultured in RPMI-1640 medium containing $10 \%$ foetal bovine serum (FBS), $100 \mathrm{U} / \mathrm{mL}$ penicillin and $100 \mu \mathrm{g} / \mathrm{mL}$ streptomycin. Cells were maintained in a humidified atmosphere of $5 \% \mathrm{CO}_{2}$ environment at $37^{\circ} \mathrm{C}$. Patient-derived primary cells were obtained from pleural fluids, which were collected aseptically and heparinised after informed consent and after approval by the Ethics Committee of the Faculty of Medicine, Chulalongkorn University (IRB 365/62).
Lentiviral production and CRISPR/Cas9-mediated gene knockdown Lentiviral plasmids carrying guided RNA (gRNA) sequence against human TRPM7 and SpCas9-blasticidin resistance were kind gifts of Profs. John Doench and David Root (Addgene \#76111) and Prof. Feng Zhang (Addgene \#52962). ${ }^{22,23}$ All-in-one lentiviral plasmids carrying SpCas9-puromycin resistance and gRNA sequence against human ORAI1, STIM1 and OGT were from GenScript (Piscataway, NJ). Lentivirus production was performed using HEK293T packaging cells (ATCC) in conjunction with pCMV.dR8.2 dvpr lentiviral packaging and pCMV-VSV-G envelope plasmids (Addgene \#8454 and 8455). ${ }^{24}$ Cells were incubated with lentiviral particles in the presence of hexadimethrine bromide for $48 \mathrm{~h}$ and the transfected cells were treated with blasticidin $(10 \mu \mathrm{g} / \mathrm{mL})$ or puromycin $(1 \mu \mathrm{g} / \mathrm{mL})$ for 3 weeks and analysed prior to use by Western blotting.

\section{Pharmacological inhibition of TRPM7}

To study the dose-response pattern of TRPM7 inhibition, pharmacological inhibition of TRPM7 was performed in conjunction with CRISPR/Cas9-mediated genetic manipulation of TRPM7 using 2-aminoethyl diphenylborinate (2-APB). 2-APB is a general TRP channel blocker that has been shown to inhibit TRPM7 currents in a dose-dependent manner, ${ }^{25-27}$ although this inhibitor is not only specific to TRPM7.

\section{Short hairpin RNA-mediated gene knockdown}

Lentiviral plasmid carrying short hairpin RNA (shRNA) sequence against human CAV1 was obtained from Santa Cruz Biotechnology, Inc. (Dallas, TX), while retroviral plasmid carrying shRNA sequences against human MYC was a kind gift from Prof. Martin Eilers (Addgene \#29435). ${ }^{28}$ Retrovirus production was performed using Platinum-A packaging cell lines (Cell Biolabs, San Diego, CA), while lentivirus production and viral particle incubation were performed as described above.

Cell migration and invasion assays

Cell migration was determined by wound healing assay. A monolayer of cells was cultured in 24-well plate and then a wound space was made with a 1-mm tip width and allowed to migrate for 24-48 h. Micrographs were taken under a phasecontrast microscope (Eclipse Ti-U with NiS-Elements, Nikon, Tokyo, Japan) and wound spaces were measured. Quantitative analysis of cell migration was performed as previously described. ${ }^{29}$ Invasion assay was performed using a 24-well transwell unit with polycarbonate filters (8- $\mu \mathrm{m}$ pore size) coated with Matrigel (BD Bioscience, San Jose, CA). Without Matrigel coating, the control inserts were used for migration assay as an alternative to the wound healing assay. Filling the lower chamber with normal growth medium containing $10 \%$ FBS, tested cells were seeded into the upper chamber in serum-free medium and incubated for $48 \mathrm{~h}$. Non-invading/migrating cells were removed from the upper side of membrane using a cotton swab and cells that invaded/ migrated to the underside were stained with $10 \mu \mathrm{g} / \mathrm{mL}$ Hoechst 33342 for $30 \mathrm{~min}$. Inserts were visualised and scored under a fluorescence microscope (Eclipse Ti-U). The raw data for wound healing and transwell assays can be found in Supplementary Tables S1 and S2.

Intracellular $\mathrm{Ca}^{2+}$ detection

Intracellular $\mathrm{Ca}^{2+}$ was determined using Fura-2 acetoxymethyl ester (Fura-2 AM) as a selective fluorescent probe. Cells were loaded with Fura-2 AM $(4 \mu \mathrm{M})$ in Hank's buffered solution (HBSS) solution containing probenecid $(2.5 \mathrm{mM})$, EGTA $(3 \mathrm{mM})$ and $0.1 \%$ bovine serum albumin at $37^{\circ} \mathrm{C}$ for $90 \mathrm{~min}$. The cells were then centrifuged, resuspended in $\mathrm{HBSS}$ and $\mathrm{CaCl}_{2}(4 \mu \mathrm{M})$ were added for $10 \mathrm{~min}$, followed by an analysis of fluorescence intensity using BD FACSCanto flow cytometry (BD Biosciences, San Jose, CA) at 340-nm excitation and 510-nm emission. 
Western blot analysis

After specific treatments, cells were incubated in a commercial lysis buffer (Cell Signaling Technology, Beverly, MA) and a protease inhibitor mixture (Roche Molecular Biochemicals, Indianapolis, IN) at $4{ }^{\circ} \mathrm{C}$ for $30 \mathrm{~min}$. Protein content was analysed using BCA protein assay (Pierce Biotechnology, Rockford, IL) and $50 \mu \mathrm{g}$ of proteins were resolved under denaturing conditions by sodium dodecyl sulfate-polyacrylamide gel electrophoresis (SDS-PAGE) and transferred onto polyvinylidene fluoride membranes. Membranes were blocked with 5\% non-fat dry milk, incubated with appropriate primary antibodies at $4{ }^{\circ} \mathrm{C}$ overnight and subsequently incubated with peroxidase-conjugated secondary antibodies for $1 \mathrm{~h}$ at room temperature. The immune complexes were analysed by enhanced chemiluminescence detection system on a digital imager (ImageQuant LAS, GE Healthcare, Pittsburgh, PA).

Co-immunoprecipitation, ubiquitination and O-GlcNAcylation Specific protein was immunoprecipitated using protein Gconjugated agarose beads (Santa Cruz Biotechnology). Briefly, cell lysates $(200 \mu \mathrm{g}$ protein) were incubated with anti-c-Myc or antiCav-1 antibody at $4{ }^{\circ} \mathrm{C}$ overnight, followed by a 3-h incubation with agarose beads at $4{ }^{\circ} \mathrm{C}$. The immune complexes were washed five times with cold lysis buffer and resuspended in $2 \times$ Laemmli sample buffer. They were then separated by SDS-PAGE and analysed for ubiquitination or O-GlcNAcylation using an antiubiquitin or anti-O-GIcNAc (RL2) antibody.

Xenograft mouse model for experimental lung metastasis Animal care and experimental procedures described in this study were performed in accordance with the Guidelines for Animal Experiments at West Virginia University (WVU) with the approval of the Institutional Animal Care and Use Committee (\#1702005551). Male NOD/SCID gamma mice, strain NOD.CgPrkdc $^{\text {scid }} \| 2 \mathrm{rg}^{\text {tmiWjl }} / \mathrm{SzJ}$ (NSG; WVU Transgenic Animal Core Facility, Morgantown, WV), aged 6-8 weeks, median weight $26 \mathrm{~g}$, were maintained under pathogen-free conditions within the institutional animal facility and assigned randomly into two experimental groups. Food and tap water were given ad libitum. A total of $1 \times 10^{6}$ cells labelled with UBC-RFP-T2A-Luciferase dual reporter were injected into mice (five mice per group) via tail vein and mice were inspected daily for any signs of distress. Tumour growth of luciferase-labelled cells was monitored at the time of inoculation (week 0; baseline) and on a weekly basis by using in vivo IVIS imaging (PerkinElmer, Waltham, MA) (see Supplementary Materials for full descriptions). At the end of experiments, mice were euthanised with cervical dislocation under carbon dioxide inhalation and the lungs, liver and other organs were dissected and further analysed for tumour histopathology.

Tumour histopathology

Isolated lung tissues from tumour-bearing mice were formalin fixed and paraffin embedded. The specimens were cut into $5-\mu \mathrm{m}$ sections and stained with haematoxylin and eosin to define the tumour morphology and cellular structure within the lungs. Tissue sectioning and haematoxylin and eosin staining were performed at the WVU Pathology Laboratory for Translational Medicine, while immunohistochemical (IHC) staining was performed at the Immunopathology Laboratory, Faculty of Medicine Siriraj Hospital.

Statistical analysis

The data represent means \pm s.d. from three or more independent experiments as indicated. Statistical analysis was performed by two-sided Student's $t$ test or one-way analysis of variance, followed by a Bonferroni post-test at a significance level of $p<0.05$.

\section{RESULTS}

Higher TRPM7 expression associates to poorer prognosis of lung carcinoma patients

Altered calcium signalling often involves an aberrant expression, cellular localisation and function of $\mathrm{Ca}^{2+}$ channels, contributing towards specific hallmarks of cancers, such as uncontrolled growth and resisting cell death. ${ }^{4,6}$ We first performed survival analysis in human NSCLC patients using Kaplan-Meier plotter based on mRNA expression of TRPM7, ORAI1 and STIM1 to assess the clinical significance of these major channels. The population of patients in the database was divided into high and low expression groups using median value to set the cut-off. Figure 1a reveals that the overall survival was significantly reduced in patients with high TRPM7 and ORAI1 expression, but not STIM1, in comparison with patients with low expression (hazard ratio $(\mathrm{HR})=1.91$ and $\mathrm{HR}=$ 1.32 , respectively), suggesting that TRPM7 and Orai1 may serve as a prognostic factor in lung carcinoma. We hypothesised that TRPM7 and Orai1 may be involved in the regulation of lung cancer metastasis. CRISPR/Cas9 system was used to inhibit TRPM7 and Orai1 in NSCLC NCl-H292 cells and cell invasion, which is a critical step in the metastatic process, was determined by the transwell assay. TRPM7i, and to a lesser extent Orai1 (ORAI1i) and STIM1 (STIM1i), reduced the invasiveness of $\mathrm{NCl}-\mathrm{H} 292$ cells as compared to CRISPR/Cas9 control (CTLi) (Fig. 1b, c). The inhibitory role of TRPM7 inhibition on cell invasion was similarly observed in $\mathrm{NCl}$ $\mathrm{H} 460, \mathrm{~A} 549$ and $\mathrm{NCl}-\mathrm{H} 23$ cells (Fig. 1d and Supplementary Fig. S1), suggesting the generality of the observed effect. Bioinformatics analysis of an association between TRPM7 expression and staging of human lung carcinoma revealed that high TRPM7 correlates well with lymph node metastasis (stage N0 versus N2) (Fig. 1e) and thus further support the role of TRPM7 in the progression of lung cancer.

Inhibition of TRPM7 and $\mathrm{Ca}^{2+}$ influx suppresses cell migration and invasion

To determine further the causal relationship between TRPM7 function and lung carcinoma cell motility, NSCLC NCl-H292 and $\mathrm{NCl}-\mathrm{H} 460$ cells were incubated with various concentrations of a non-specific inhibitor of TRPM7, 2-APB, and its effects on $\mathrm{Ca}^{2+}$ influx, cell migration and invasion were evaluated. We identified and used the appropriate noncytotoxic concentrations of 2-APB which is up to $25 \mu \mathrm{M}$, to ensure that the observed effects are not due to the cytotoxicity or reduced proliferation. Free intracellular $\mathrm{Ca}^{2+}$ in response to 2-APB treatment was evaluated by flow cytometry using Fura-2 $\mathrm{AM}$ as a specific fluorescence probe. Figure $2 a$ shows that 2-APB $(10-25 \mu \mathrm{M})$ was able to decrease the fluorescence intensity of $\mathrm{Ca}^{2+}$-bound Fura- 2 under the control level, thus validating its inhibitory effect on $\mathrm{Ca}^{2+}$ influx (see also Supplementary Fig. S2). Treatment of $\mathrm{NCl}-\mathrm{H} 292$ cells with 2-APB $(0-25 \mu \mathrm{M})$ caused a dose-dependent decrease in cell invasion (Fig. 2b and Supplementary Fig. S3), in agreement with the results observed from TRPM7 gene manipulation (Fig. 1b). Likewise, cell migration, as evaluated by both transwell and wound healing assays, was dose-dependently decreased by the 2-APB treatment $(0-25 \mu \mathrm{M})$ in NSCLC cells (Fig. $2 \mathrm{c}-\mathrm{f}$ and Supplementay Figs. S3 and S4).

Lung cancer patient-derived primary cells were obtained from fresh patient-derived pleural effusion (see Supplementary Table S3 for their clinical characterisation) and similarly evaluated for cell migration by wound healing assay following 2-APB treatment $(0-25 \mu \mathrm{M})$. Consistent with the findings obtained from NSCLC cell lines, we observed a dose-dependent decrease in cell migration upon TRPM7 inhibition in multiple primary lung cancer cells (Fig. $2 \mathrm{~g}$ and Supplementary Fig. S5). Together, these results demonstrate the role of TRPM7 and $\mathrm{Ca}^{2+}$ influx in NSCLC cell motility regulation. 
a
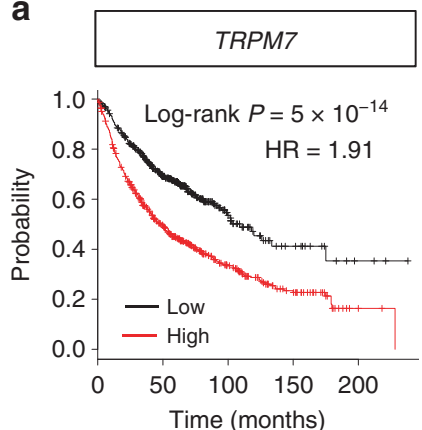

b
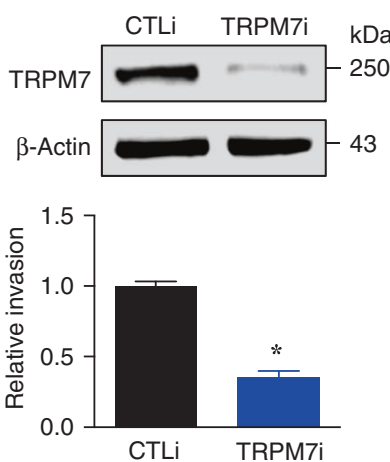
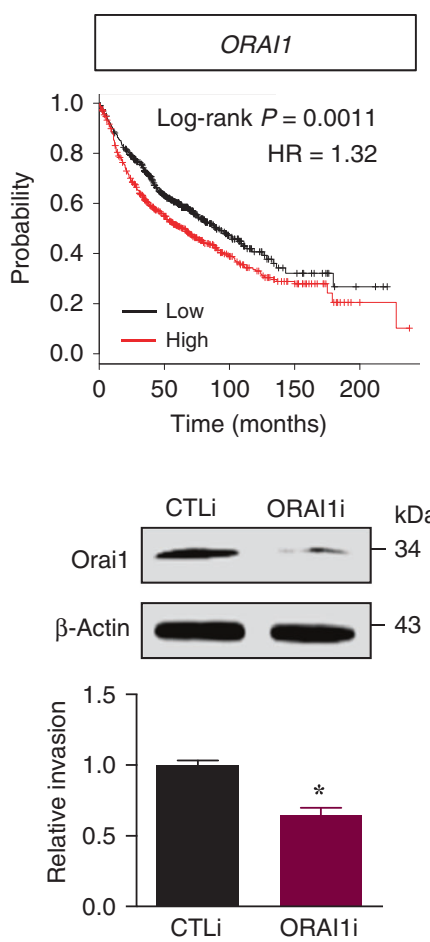
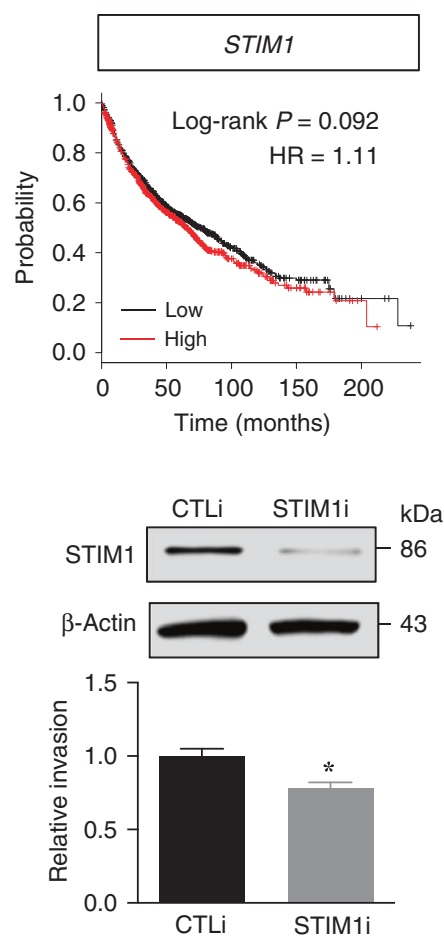

C
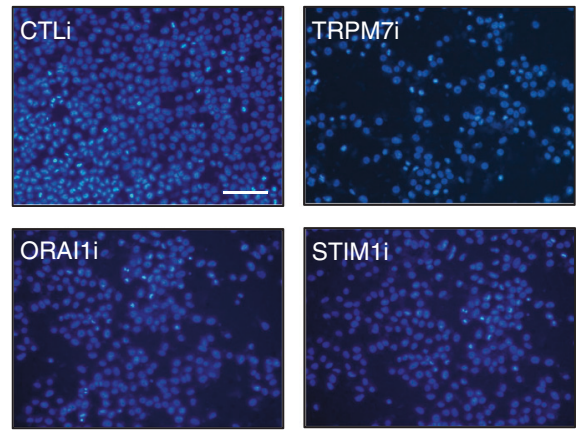

\section{STIM1i}

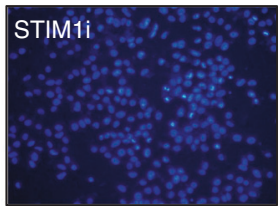

d
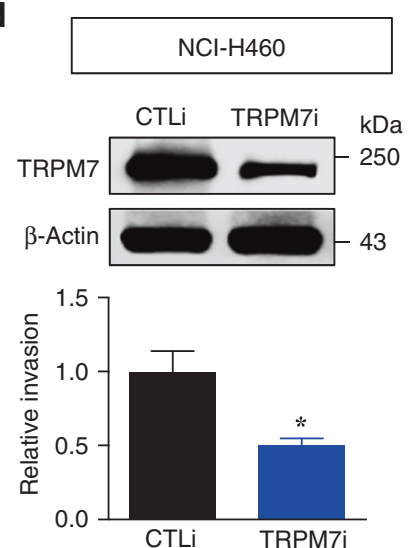
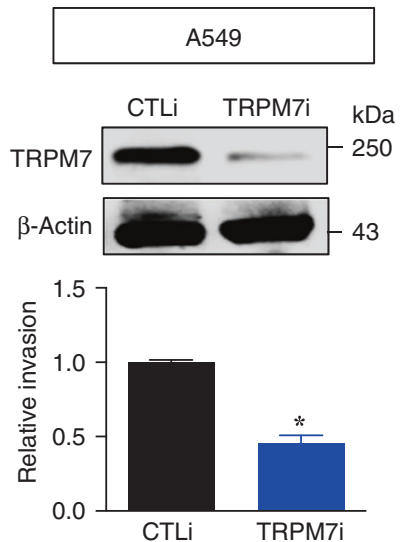

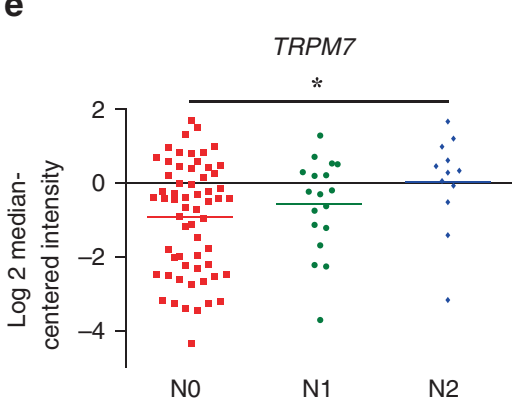

No
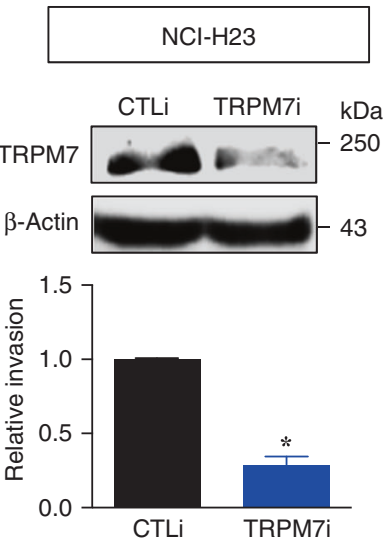

TRPM7 regulates cell motility mainly through O-GIcNAcylation Hyper-O-GIcNAcylation is a general feature of various human malignancies that may contribute to transformed phenotypes. Level of $O$-GlcNAcylation has been suggested to be a regulator of cancer metastasis in human prostate, breast and ovarian cancers. ${ }^{30-32}$ We hypothesised a plausible crosstalk between the calcium signalling and O-GIcNAcylation and herein tested this possibility in NSCLC. We observed a remarkable decrease in global O-GlcNAcylation in NSCLC cells upon treatment with the TRPM7 inhibitor 2-APB $(>10 \mu \mathrm{M})$ (Fig. 3a) and upon TRPM7 knockdown (Fig. 3b), suggesting the functional linkage between TRPM7mediated cell motility and $O$-GlcNAcylation.

$O$-GlcNAcylation is dynamically modulated by two cycling enzymes O-GlcNAc transferase (OGT) and O-GlcNAcase (OGA) 
Fig. 1 TRPM7 expression is correlated to poor prognosis and progression of NSCLC. a Kaplan-Meier survival plots of NSCLC patients from the gene microarrays generated using the Kaplan-Meier plotter according to the level of TRPM7, ORAI1 and STIM1. Overall survival of patients with highest expression (cut-off: median, red line) is compared to those with lowest expression (black line) $(n=2437)$. b, c NCl-H292 cells were genetically knocked down with TRPM7 (TRPM7i), Orai1 (ORAI1i), STIM1 (STIM1i) or control (CTLi) gRNAs in the CRISPR/Cas9 system. b (Upper) TRPM7, Orai1 and STIM1 levels were analysed by Western blotting. (Lower) Cell invasion was evaluated by the transwell assay, where the penetrating cells were stained by Hoechst 33342 dye, quantified and reported as a ratio to CTLi cells. Data are mean \pm s.d. $(n=4)$. ${ }^{*} p<0.05$ versus CTLi cells; two-sided Student's $t$ test (see also Supplementary Table S1 for raw data). c Representative fluorescence micrographs of invading cells stained with Hoechst 33342 dye are shown. Scale bar $=100 \mu \mathrm{m}$. d Analysis of cell invasion in TRPM7 knockdown (TRPM7i) and control (CTLi) NCl-H460, A549 and $\mathrm{NCl}-\mathrm{H} 23$ cells by the transwell assay. (Upper) Western blot analysis of TRPM7 level. (Lower) Quantitative analysis of invading cells stained with Hoechst 33342 dye (see also Supplementary Fig. S1 for additional micrographs). Data are mean \pm s.d. $(n=4) .{ }^{*} p<0.05$ versus CTLi cells; two-sided Student's $t$ test. e Differential expression of TRPM7 in normal and lung carcinoma tissues in Bittner's dataset according to their TNM staging $(n=109) .{ }^{*} p<0.05$ versus N0; two-sided Student's $t$ test.

that catalyses and removes $O$-GlcNAc. We next manipulated OGT in NCl-H292 cells by CRISPR/Cas9-mediated gene knockdown to decrease cellular $O$-GlcNAcylation to ascertain its role in cell motility of NSCLC cells. Similar to that observed in TRPM7i cells (Fig. 3c, d), an inhibition of OGT effectively decreased cell migration as compared to control (Fig. 3e, $\mathrm{f}$ and Supplementary Fig. S6), thus confirming the promoting role of hyper-OGlcNAcylation in NSCLC motility. The significance of hyper-OGlcNAcylation was further strengthened by an observed low MGEA5 (encoding OGA) expression in clinical lung tumour specimens as compared to normal lung tissues (Fig. $3 \mathrm{~g}$ ) that were also found to be associated with lower survival rates of patients (Supplementary Fig. S7).

To further validate that TRPM7 regulates cell motility through O-GIcNAcylation, rescue experiments were conducted in which $O$-GlcNAcylation was induced in TRPM7 knockdown cells and its effects on cell motility and $\mathrm{Ca}^{2+}$ influx were examined. Thiamet $\mathrm{G}$, a small-molecule inhibitor of OGA, was used to induce cellular $O$-GIcNAcylation in this study. Intracellular $\mathrm{Ca}^{2+}$ level was found to be reduced in TRPM7i cells, but not further changed in thiamet G-treated cells in both TRPM7i and CTLi cells (Fig. 3h; see also Supplementary Fig. S8). Figure 4a shows that although thiamet $G$ had minimal effect on the nontreated control cells, which is likely due to their limited migratory capacity, cotreatment of thiamet $\mathrm{G}(10 \mu \mathrm{M})$ and 2-APB $(20 \mu \mathrm{M})$ significantly promoted cell migration as compared to 2-APB treatment alone. A similar finding on the rescue of TRPM7i cell migration by thiamet $\mathrm{G}(10 \mu \mathrm{M})$ was also observed (Fig. 4b). Altogether, these data indicate that $O$-GICNAcylation is a downstream regulator of TRPM7 and $\mathrm{Ca}^{2+}$ influx and is involved in cell motility regulation in NSCLC cells.

Cav-1 and c-Myc are favourable downstream targets of TRPM7/OGIcNAc

To elucidate the underlying mechanisms by which TRPM7 and O-GlcNAcylation mediate cell motility, we monitored the levels of various proteins known to be important in metastasis of lung tumour, including matrix metalloproteinases MMP-2 and MMP$9,{ }^{33,34}$ SOX9, ${ }^{35}$ C-Myc and Cav-1, ${ }^{36,37}$ following TRPM7 inhibition. The results show that MMP-2, MMP-9 and SOX9 were relatively unchanged when comparing TRPM7i and control cells, suggesting their unlikely involvement in the TRPM7/O-GICNAc regulatory axis (Fig. 4c). On the other hand, a striking downregulation of c-Myc and Cav-1 was observed in TRPM7i cells (Fig. 4c, $d$ and Supplementary Fig. S9) as well as in 2-APB-treated primary cells (Fig. 4e), suggesting C-Myc and Cav-1 as potential molecular targets of this regulatory process. It is worth noting here that the precise mechanism of how TRPM7 regulates O-GIcNAcylation remains unclear and is likely to be complex and diverse since $O$-GlcNAcylation senses and responds to a wide variety of stimuli and stresses. However, our results indicate that this might involve a modulation of O-GIcNAc cycling enzymes, as an increase of OGA and an upregulation of OGT were observed in the TRPM7i cells (Supplementary Fig. S9).
TRPM7 and O-GlcNAcylation inhibit Cav-1 and c-Myc degradation To gain a better insight into how TRPM7 modulates Cav-1 and cMyc, quantitative real-time PCR was performed to analyse their mRNA expression in TRPM7i and control cells. Supplementary Fig. S9 shows that TRPM7 inhibition had no significant effect on CAV1 transcription, suggesting that Cav-1 is regulated by TRPM7 at post-translational level. By contrast, a subtle yet significant downregulation of MYC was observed in TRPM7i cells, despite a large decrease in c-Myc protein. It is hence conceivable that TRPM7 regulates c-Myc at both transcriptional and posttranslational levels.

O-GlcNAcylation is known to influence protein stability and function. ${ }^{21,38,39}$ To directly determine whether O-GlcNAcylation of c-Myc and Cav-1 could protect them from TRPM7 inhibition, TRPM7i cells were treated with thiamet $G(10 \mu \mathrm{M})$ that causes hyper-O-GlcNAcylation (Supplementary Fig. S10) and its effect on Cav- 1 and c-Myc was evaluated by Western blotting. Figure $4 \mathrm{f}$ and Supplementary Fig. S11a shows that thiamet $G$ reversed the repression of Cav-1 and c-Myc by TRPM7 inhibition in TRPM7i cells, thereby confirming the TRPM7/O-GICNAc regulatory axis of Cav-1 and c-Myc. Proteasomal degradation is a major post-translational cellular process that controls protein turnover. To determine whether Cav-1 and c-Myc under TRPM7 regulation are controlled by proteasomal degradation, TRPM7i cells were treated with MG132 for proteasome inhibition. The increase in Cav-1 and c-Myc expression by MG132 treatment suggested proteasomal degradation as an important mechanism of Cav- 1 and c-Myc regulation by TRPM7 and $\mathrm{O}-$ GlcNAcylation (Fig. $4 \mathrm{~g}$ and Supplementary Fig. $\mathrm{S} 11 \mathrm{~b})$. To strengthen this finding, the dose profile of Cav-1 and c-Myc repression in response to 2-APB treatment $(0-25 \mu \mathrm{M})$, which was substantially rescued by the presence of thiamet $G(10 \mu \mathrm{M})$, was generated and shown in Fig. 5 a.

Given that numerous proteins, including Cav-1 and c-Myc, are marked for proteasomal degradation by ubiquitination, ${ }^{21,29}$ we analysed Cav- 1 and c-Myc ubiquitination in TRPM7i and CTLi cells in the presence or absence of thiamet $\mathrm{G}(10 \mu \mathrm{M})$. Figure $5 \mathrm{~b}$ shows that TRPM7 inhibition induced Cav-1 and C-Myc ubiquitination and that an addition of thiamet $\mathrm{G}$ could inhibit their ubiquitination (see also Supplementary Fig. S12). As both O-GIcNAcylation and ubiquitination occurs on serine and/or threonine residues of protein, it is plausible that $O$-GlcNAcylation might interfere with Cav-1 and c-Myc ubiquitination and subsequently protect them from proteasomal degradation. Co-immunoprecipitation of Cav-1 and c-Myc with O-GlcNAc in $\mathrm{NCl}-\mathrm{H} 292$ and $\mathrm{NCl}-\mathrm{H} 460$ cells confirms that Cav-1 and c-Myc are true targets of O-GlcNAcylation in NSCLC (Fig. 5c, d). Phenotypically, hyper-O-GlcNAcylation by thiamet $\mathrm{G}$ was demonstrated to reactivate the migration of $\mathrm{NCl}-\mathrm{H} 292$ cells following Cav-1 and c-Myc inhibition by shRNA-mediated gene knockdown in agreement with the results of Cav-1, c-Myc and O-GlcNAc levels (Fig. 5e-i and Supplementary Fig. S13). Rescue experiments with Cav-1 and c-Myc overexpression were also conducted in TRPM7i cells (Supplementary Fig. S14) to once again validate that Cav-1 and c-Myc are regulated by TRPM7/O-GICNAc axis. 
a 2-APB

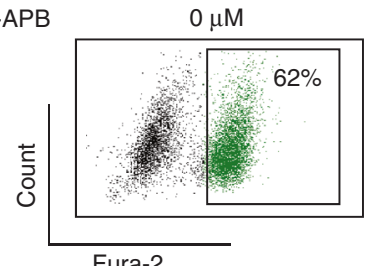

Fura-2

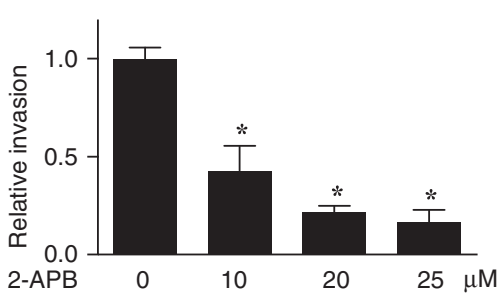

d
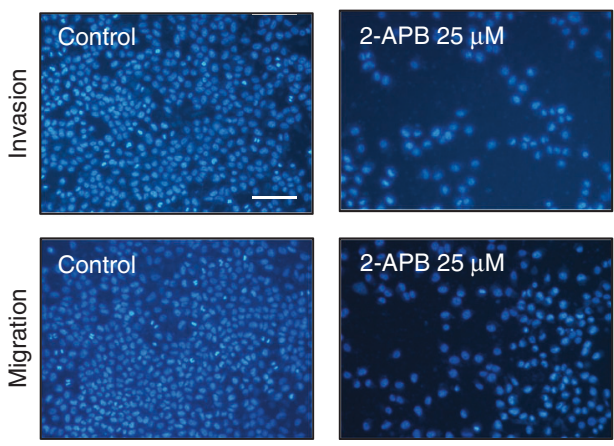

e

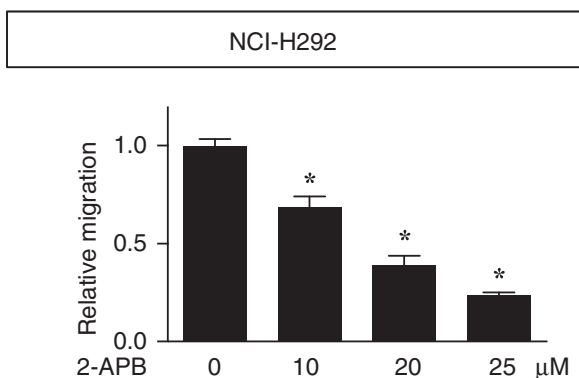

$48 \mathrm{~h}$
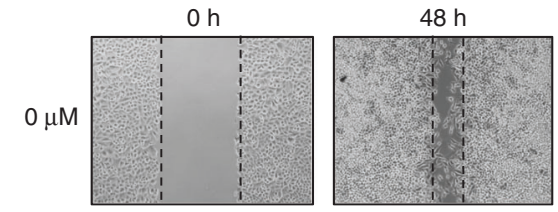

$25 \mu \mathrm{M}$
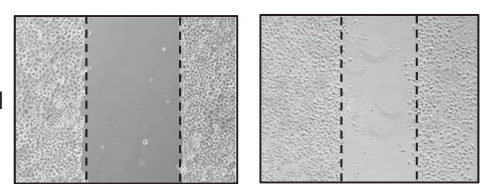

C

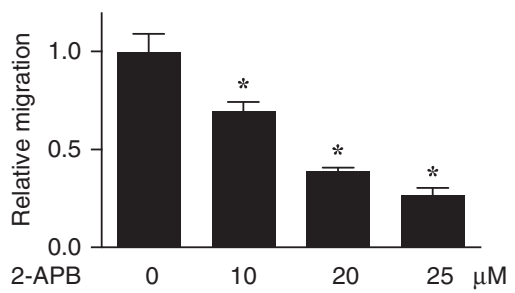

f
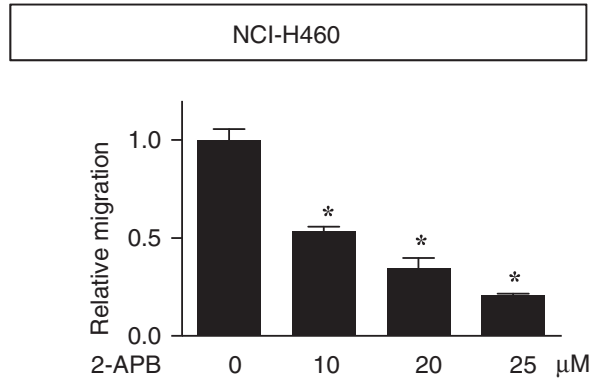

g
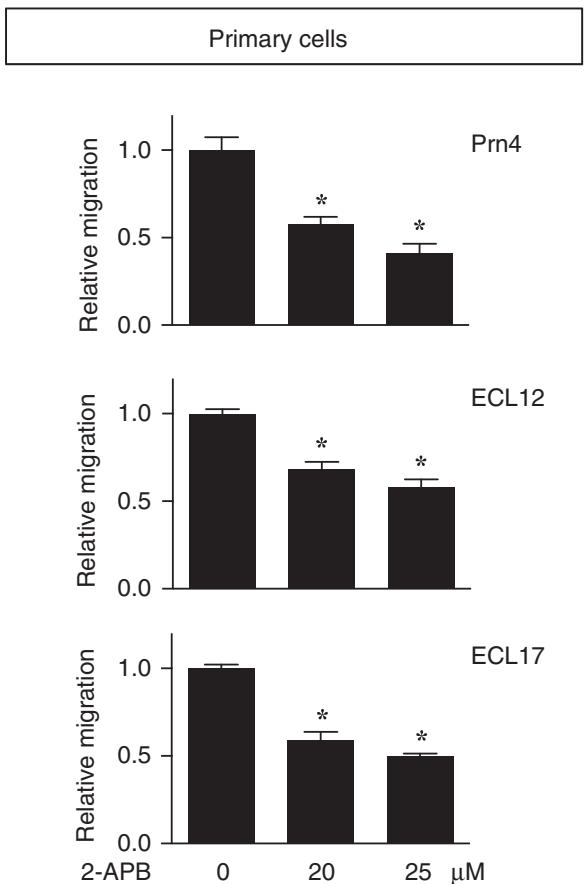

Fig. 2 Inhibition of TRPM7 suppresses NSCLC cell migration and invasion. a Flow cytometric analysis of intracellular Ca ${ }^{2+}$ in $\mathrm{NCI}^{-\mathrm{H} 292}$ cells treated with TRPM7 inhibitor 2-APB $(0-25 \mu \mathrm{M})$ using Fura-2 AM as a specific fluorescent probe (see also Supplementary Fig. S2 for quantitative analysis of intracellular $\mathrm{Ca}^{2+}$ by a fluorescence plate reader). b-d NCl-H292 and NCI-H460 cells were treated with $2-\mathrm{APB}(0-25 \mu \mathrm{M})$ and cell invasion (b) and migration (c) were evaluated by transwell assays at $48 \mathrm{~h}$ post incubation. Data are mean $\pm s . d$. $(n=4)$. ${ }^{*} p<0.05$ versus nontreated cells; two-sided Student's $t$ test. d Representative fluorescence micrographs of migrating and invading cells stained with Hoechst 33342 dye are shown. Scale bar $=100 \mu \mathrm{m}$. e-g 2-APB-treated NCl-H292 (e), NCl-H460 (f) and primary lung cancer (g) cells were evaluated for cell migration by wound healing assay. Wound space was visualised at 0 and 24 or $48 \mathrm{~h}$ and \% change in the space was calculated and reported as a ratio to nontreated cells. Data are mean \pm s.d. $(n=4) .{ }^{*} p<0.05$ versus nontreated cells; two-sided Student's $t$ test (see also Supplementary Table S2 for raw data). 
a
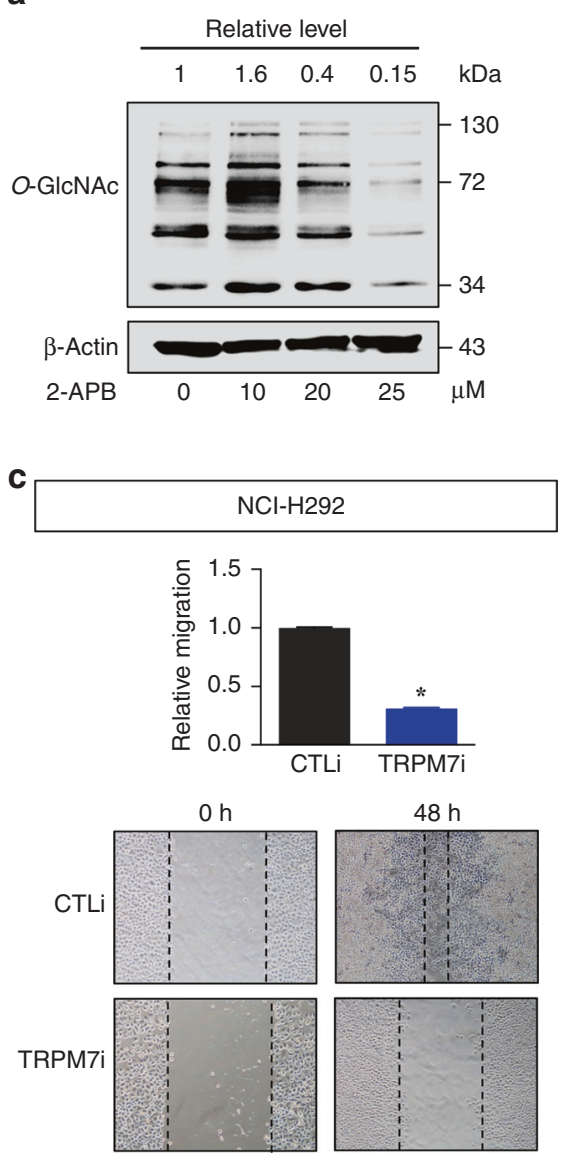

e

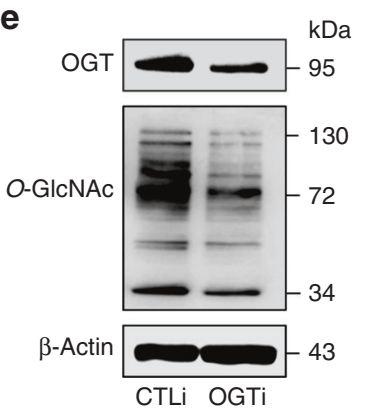

$\mathbf{h}$

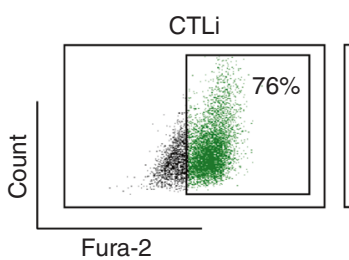

b

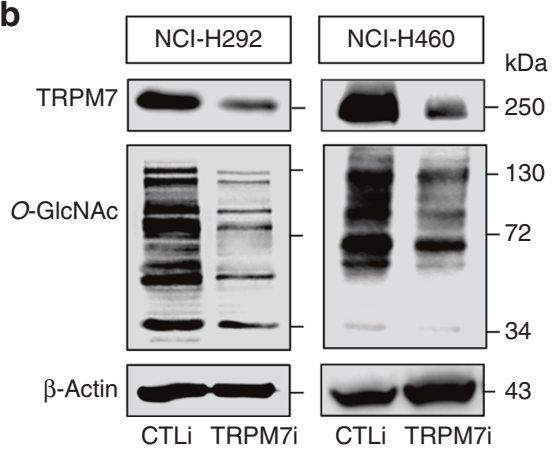

d
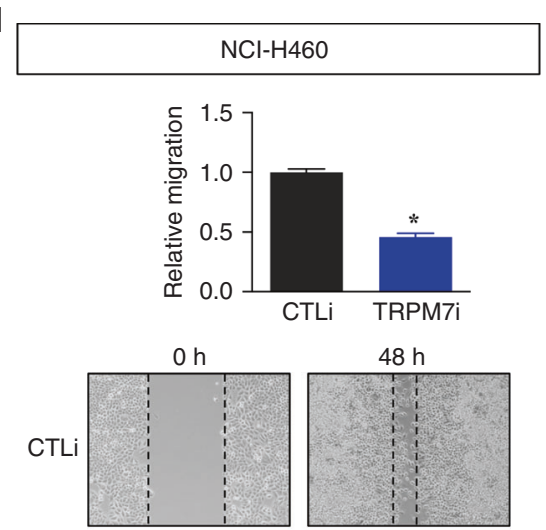

$48 \mathrm{~h}$
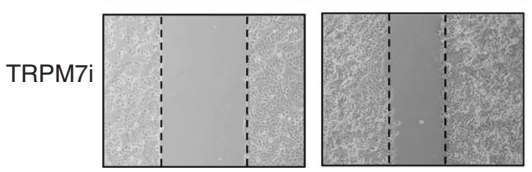

g

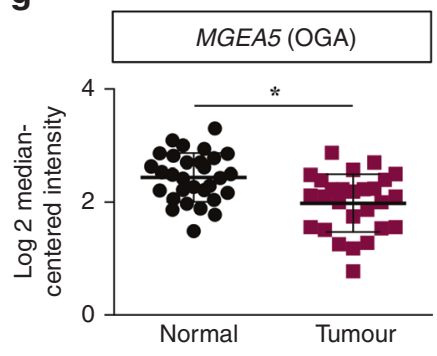

Fig. 3 TRPM7 regulates O-GIcNAcylation in NSCLC cells. a Western blot analysis of global O-GlcNAc level in NCl-H292 cells treated with 2 APB (0-25 $\mu \mathrm{M})$. $\beta$-Actin was used as a loading control. b Western blot analysis of TRPM7 and O-GlcNAc levels in TRPM7 knockdown (TRPM7i) $\mathrm{NCl}-\mathrm{H} 292$ and $\mathrm{NCl}-\mathrm{H} 460$ cells. c, d Cell migration of TRPM7i NCl-H292 (c) and NCl-H460 (d) cells was compared to that of CTLi cells using wound healing assay at $48 \mathrm{~h}$. Plots are relative migration calculated from $\%$ change in wound space and normalised to CTLi cells. Data are

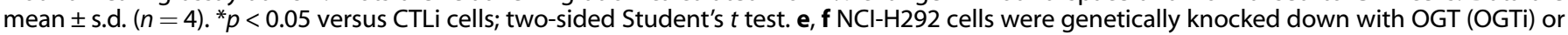
control (CTLi) gRNAs in CRISPR/Cas9 system (e) and cell migration was evaluated by wound healing assay at $48 \mathrm{~h}(\mathbf{f})$. Plots are relative migration calculated from $\%$ change in wound space and normalised to CTLi cells. Data are mean \pm s.d. $(n=4)$. ${ }^{*} p<0.05$ versus CTLi cells; twosided Student's $t$ test. Representative micrographs of wound space are shown in Supplementary Fig. S6. g Differential expression of MGEA5 (encoding OGA) in normal and lung carcinoma tissues in the Su's dataset. ${ }^{*} p<0.05$ versus CTLi cells; two-sided Student's $t$ test. See also Supplementary Fig. S7 for Kaplan-Meier survival curve of patients with NSCLC according to MGEA5 expression. $\mathbf{h}$ Flow cytometric analysis of intracellular $\mathrm{Ca}^{2+}$ in TRPM7i and CTLi cells in the presence or absence of the OGA inhibitor thiamet G (TMG; $\left.10 \mu \mathrm{M}\right)$ using Fura-2 AM as a specific fluorescent probe (see also Supplementary Fig. S8 for quantitative analysis of intracellular $\mathrm{Ca}^{2+}$ by a fluorescence plate reader). 
a
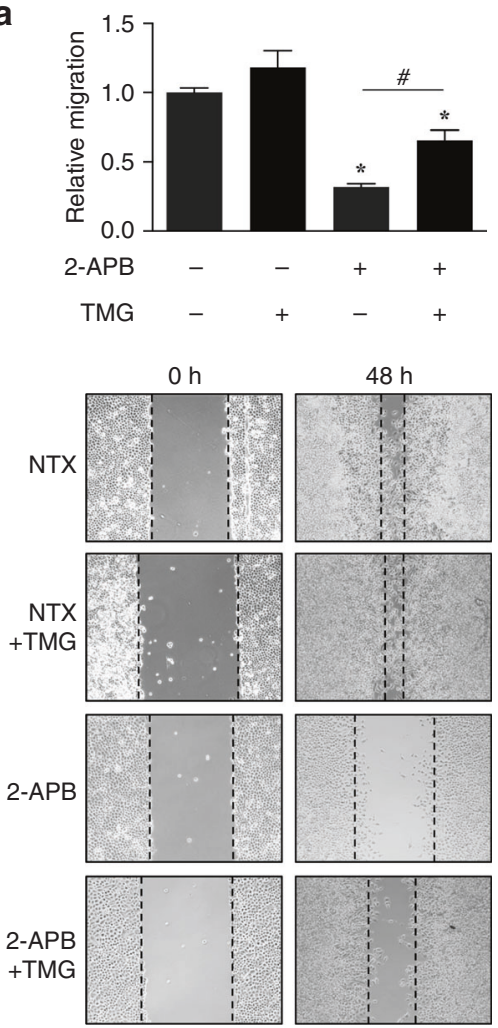

C

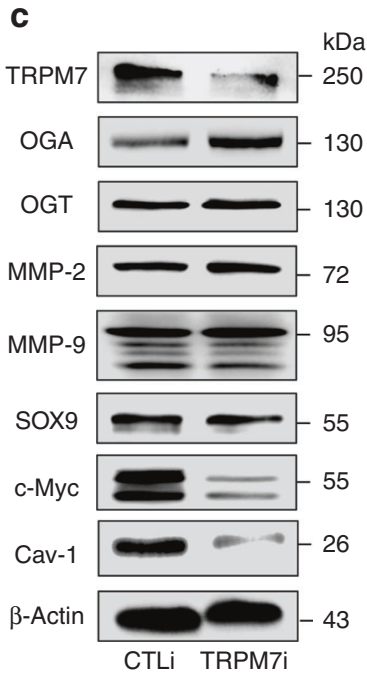

$48 \mathrm{~h}$
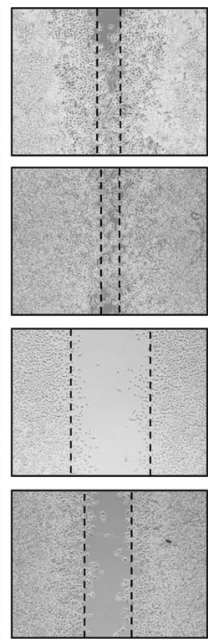

b
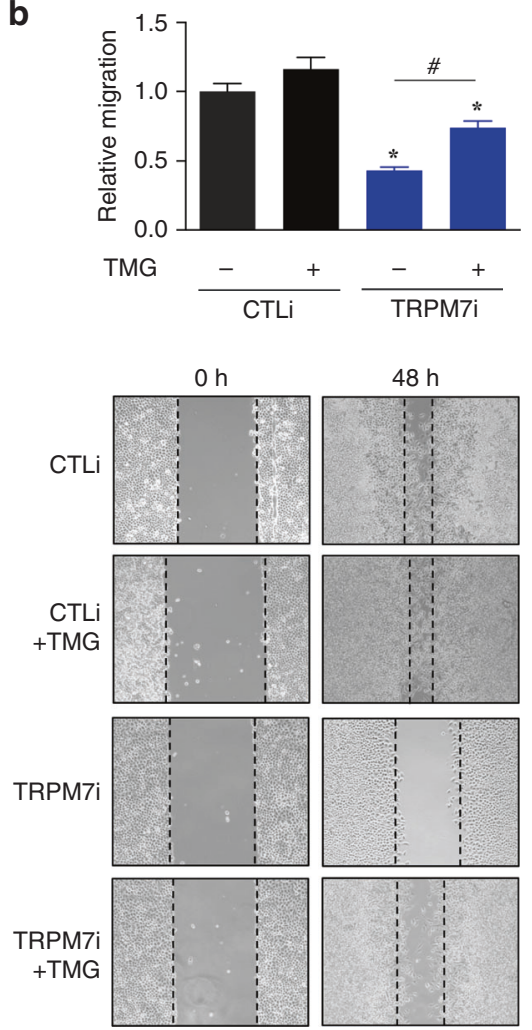

e d $=\mathrm{CTLi}$
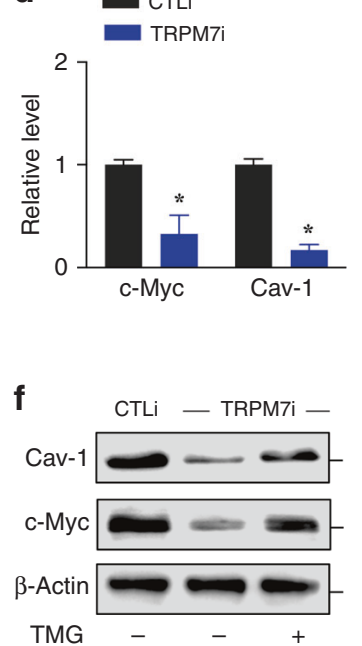

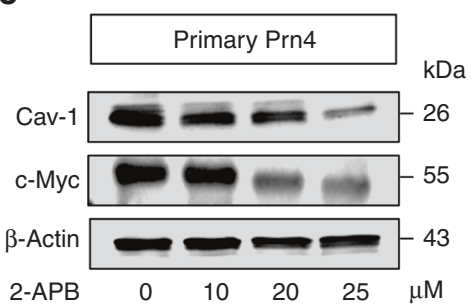

g

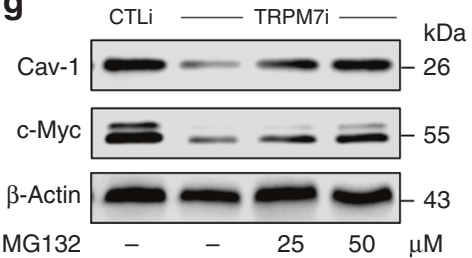

Fig. 4 NSCLC cell motility is mediated by TRPM7/O-GIcNAc regulatory axis. a, b Cell migration assay of $\mathrm{NCl}-\mathrm{H} 292$ cells upon TRPM7 inhibition by 2-APB $(20 \mu \mathrm{M})$ (a) or CRISPR/Cas9 (TRPM7i) (b) in the presence or absence of thiamet G (TMG; $10 \mu \mathrm{M})$ at $48 \mathrm{~h}$. Plots are relative migration normalised to control cells. Data are mean \pm s.d. $(n=4) .{ }^{*} p<0.05$ versus nontreated or CTLi cells; one-way ANOVA with Bonferroni post-test. ${ }^{\#} p<0.05$ versus 2-APB-treated or TRMP7i cells without TMG; one-way ANOVA with Bonferroni post-test. c Western blot analysis of TRPM7, OGA, OGT or key regulators of lung tumour metastasis, including MMP-2, MMP-9, SOX9, c-Myc and Cav-1 in TRPM7i and CTLi cells. d Quantitative analysis of c-Myc and Cav-1 by densitometry is shown (see also Supplementary Fig. S9 for quantitative analysis of other proteins and quantitative real-time PCR of TRPM7, MGEA5, OGT, MYC and CAV1 mRNA expression). Plots are fold difference relative to CTLi cells after normalisation to the loading control. Data are mean \pm s.d. $(n=3)$. ${ }^{*} p<0.05$ versus CTLi cells; two-sided Student's $t$ test. e Inhibition of TRPM7 decreases Cav-1 and c-Myc in lung cancer patient-derived primary cells. Primary Prn4 cells were treated with 2-APB (0-25 $\mu M$ ) for $24 \mathrm{~h}$ and Cav-1 and c-Myc levels were evaluated by Western blotting. $\beta$-Actin was used as a loading control. f, $\mathbf{g}$ Western blot analysis of Cav- 1 and c-Myc in TRPM7 knockdown (TRPM7i) and control (CTLi) NCl-H292 cells in the presence or absence of the OGA inhibitor thiamet G (TMG; 10 $\mu \mathrm{M})(\mathbf{f})$ or proteasomal inhibitor MG132 $(25-50 \mu \mathrm{M})(\mathbf{g})$ (see also Supplementay Fig. S11 for quantitative analysis of proteins by densitometry).

Inhibition of TRPM7 attenuates lung metastasis

Having demonstrated that TRPM7 acts upstream of O-GIcNAcylation, Cav-1 and C-Myc, regulating cell migration and invasion in NSCLC cells, we experimentally verified the involvement of TRPM7 in metastasis using a xenograft mouse model. TRPM7i and control cells were genetically labelled with luciferase and injected into NSG mice via tail vein at the dose of $1 \times 10^{6}$ cells per mouse. Tumour growth was monitored weekly by measuring the 


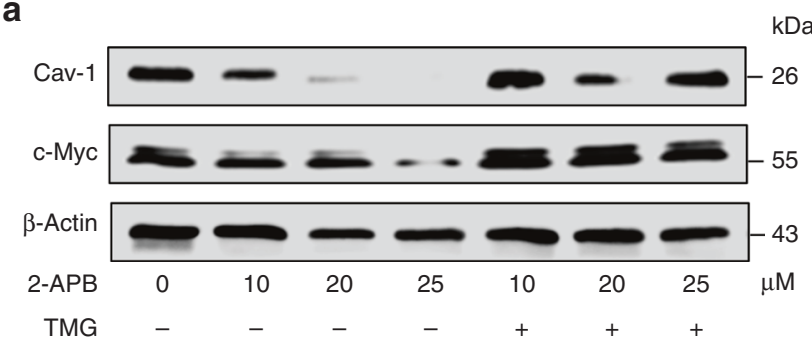

b
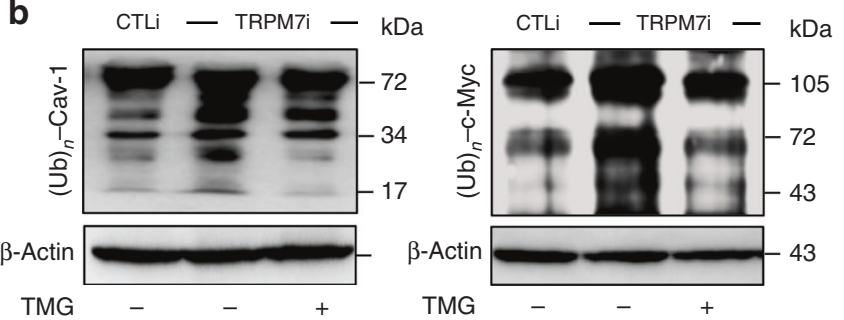

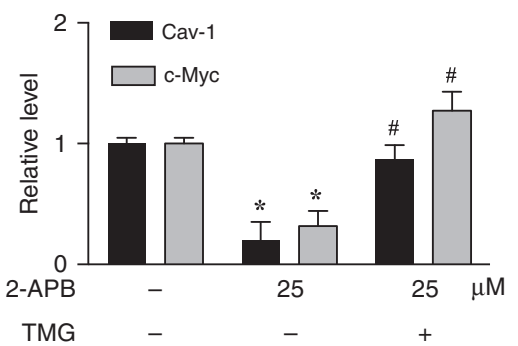

TMG

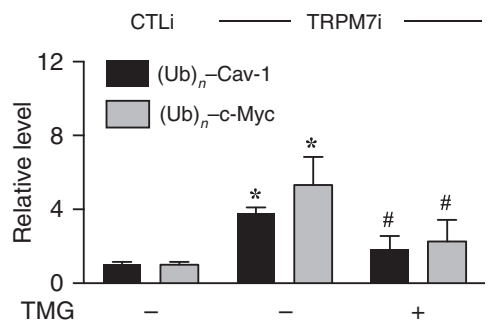

C

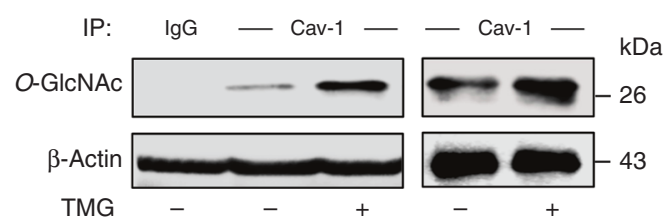

e

CTLi - CAV1i - $\mathrm{kDa}$

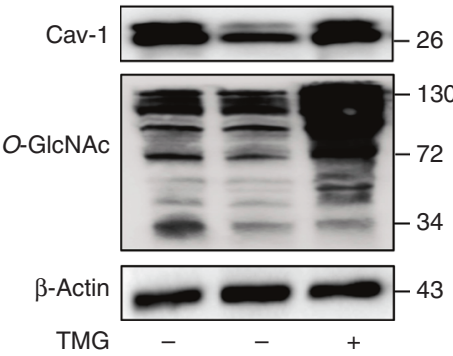

g

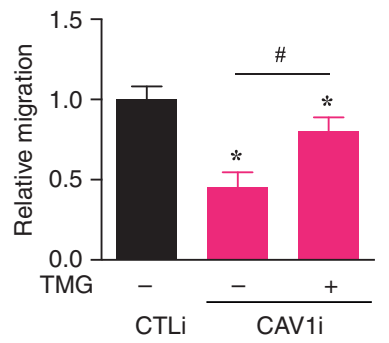

f

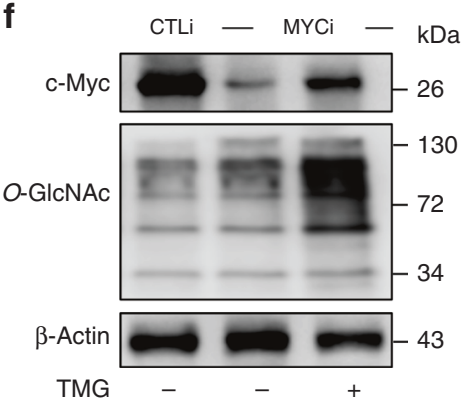

h

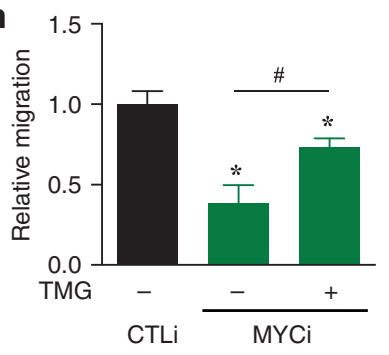

d

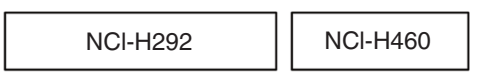

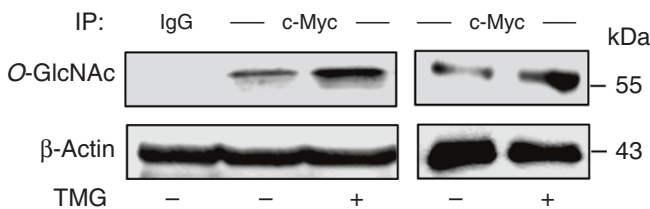

i
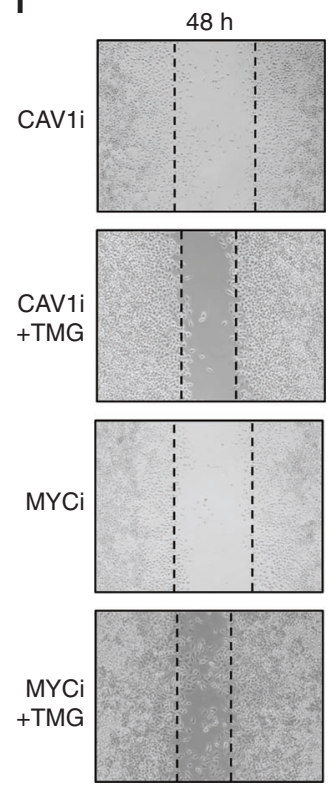

luciferase activity associated with the growing tumour cells using IVIS bioluminescence imaging (Fig. 6a). Quantitative analysis of luminescence signals revealed that tumour growth was significantly lower in mice bearing TRPM7i cells relative to control mice at week 1, 2 and 3 post injection (Fig. 6a, b), consistent with the observations of reduced number of experimental lung metastases (Fig. 6c, upper). IHC analysis further revealed the overall diminished intensities of Cav-1 and c-Myc in the isolated TRPM7i lungs (Fig. 6d), due to both lower expression and lower number of infiltrated cells, consistent with the in vitro findings (see also
Supplementary Fig. S15). It should be noted that mice were sacrificed after 3 weeks post injection because more than half of the control mice died. Anatomical and histological analyses of the dissected organs in TRPM7i mice showed much less metastatic cells in major organs, such as the liver (Fig. 6c, lower) and kidney (data not shown), as compared to control mice. These data support the in vitro findings and substantiate the critical regulatory role of TRPM7 in tumour metastasis of NSCLC and highlight the potential application of TRPM7 inhibition for metastasis of lung cancer. 
Fig. 5 Cav-1 and c-Myc are favourable targets of TRPM7/O-GIcNAc regulatory axis. a Dose profile of Cav-1 and c-Myc levels in response to 2-APB treatment $(0-25 \mu \mathrm{M})$ in the presence or absence of thiamet $\mathrm{G}(\mathrm{TMG} ; 10 \mu \mathrm{M})$, as evaluated by Western blotting. Plots are fold difference relative to nontreated control cells after normalisation to the loading control. Data are mean \pm s.d. $(n=3)$. ${ }^{*} p<0.05$ versus nontreated cells; one-way ANOVA with Bonferroni post-test. ${ }^{\#} p<0.05$ versus 2-APB-treated cells without TMG; one-way ANOVA with Bonferroni post-test. b Analysis of Cav-1 and c-Myc ubiquitination in TRPM7i and CTLi cells in the presence or absence of TMG (10 $\mu \mathrm{M})$ at $3 \mathrm{~h}$. All cells were treated with MG132 $(50 \mu \mathrm{M})$ to prevent proteasomal degradation of Cav-1 and c-Myc and cell lysates were prepared and immunoprecipitated using anti-Cav-1 (left) and anti-c-Myc (right) antibodies. $\beta$-Actin of IP input lysate was used as a loading control. Data are mean \pm s.d. $(n=3)$. ${ }^{*} p<0.05$ versus CTLi cells; one-way ANOVA with Bonferroni post-test. ${ }^{\#} p<0.05$ versus TRPM7i cells without TMG; one-way ANOVA with Bonferroni posttest. The efficiency of Cav-1 and c-Myc immunoprecipitation was shown in Supplementary Fig. S12. c, d Analysis of Cav-1 and c-Myc OGlcNAcylation in NCl-H292 or NCl-H460 cells to confirm that Cav-1 and c-Myc are targets of O-GlcNAcylation. Cells were treated with TMG for $6 \mathrm{~h}$ and cell lysates were prepared and immunoprecipitated using anti-Cav-1 (c) and anti-c-Myc (d) antibodies. $\beta$-Actin of IP input lysate was used as a loading control. e-i NCl-H292 cells were genetically knocked down with CAV1 (CAV1i), MYC (MYCi) or control (CTLi) shRNAs. e, $\mathbf{f}$ Western blot analysis of Cav-1, c-Myc and O-GlcNAc levels in CAV1 $i$ and MYCi cells in the presence or absence of TMG (10 $\mu$ M) for $24 \mathrm{~h}$. g-i Cell migration assay of CAV1i, MYCi and CTLi cells in the presence or absence of TMG $(10 \mu M)$ as evaluated by wound healing assay at $48 \mathrm{~h}$. Plots are relative migration calculated from $\%$ change in wound space and normalised to CTLi cells. Data are mean $\pm \mathrm{s} . \mathrm{d}$. $(n=4)$. ${ }^{*} p<0.05$ versus CTLi cells; one-way ANOVA with Bonferroni post-test. ${ }^{\#} p<0.05$ versus CAV1i or MYCi cells without TMG; one-way ANOVA with Bonferroni post-test. Representative micrographs of wound space are shown here (i) and in Supplementary Fig. S13. See also Supplementary Fig. S14 for phenotype rescue experiments upon TRPM7 inhibition by Cav-1 and c-Myc overexpression.

\section{DISCUSSION}

Dysregulation of calcium signalling is often deleterious and has been linked to certain hallmarks of cancers. However, how aberrant $\mathrm{Ca}^{2+}$ influx contributes to tumorigenesis and tumour progression, in particular metastasis in lung cancer, remains largely an unexplored area. In this study, we found that the $\mathrm{Ca}^{2+}$ permeable cation channel TRPM7, which is associated with progression and poor prognosis in lung cancer patients, is a key regulator of NSCLC cell migration and invasion and overall metastatic activity. A novel crosstalk between TRPM7 and $O$ GlcNAcylation was uncovered and their molecular targets for controlling NSCLC motility were subsequently identified as Cav-1 and c-Myc.

Virtually all cellular processes that are crucial for determining cell survival, proliferation and normal tissue homeostasis rely on ion channels. Given that aberrant expression and/or function of ion channels can impair these processes driving normal cells into malignant derivatives, the channels that support malignant behaviours of tumour cells or promote core features of cancers, which include: self-sufficiency in growth signals, insensitivity to anti-growth signals, resistance to apoptosis, limitless replication, sustained angiogenesis, and tissue invasion and metastasis; these are called oncochannels. ${ }^{40,41}$ As calcium is a universal signalling ion regulating a plethora of cellular processes, it is not surprising that numerous $\mathrm{Ca}^{2+}$ channels are being recognised as oncochannels.

The role of $\mathrm{Ca}^{2+}$ channels in facilitating malignant transformation is better known in cell proliferation. $\mathrm{Ca}^{2+}$ homeostasis has been shown to be implicated in the regulation of multiple cell cycle checkpoints, for example, cyclins A and E, with the calcium/calcineurin/NFAT pathway being one of the most recognised pathways. ${ }^{10,42}$ Relatively little is known about the role of $\mathrm{Ca}^{2+}$ channels in cell motility, which is a critical step in tumour metastasis. Previous studies have suggested the unique function of TRPM7 in controlling cell migration and invasion of ovarian, breast and prostate cancers, yet the precise underlying mechanisms are largely unknown and may vary depending on cellular context. For example, TRPM7 was reported to promote cell migration and tumour growth of prostate cancer cells through cholesterol-dependent $\mathrm{Ca}^{2+}$ entry, ${ }^{43}$ while it induces cell migration of oestrogen receptor-negative metastatic breast cancer cells through its kinase domain independent of $\mathrm{Ca}^{2+}$ influx. ${ }^{44}$ In ovarian cancer cells, TRPM7 was shown to influence the assembly of focal adhesions and Akt, Src and p38 signalling associated with cell motility. ${ }^{45}$ We further provide evidence in the present study that the role of TRPM7 in NSCLC cell migration and invasion is likely $\mathrm{Ca}^{2+}$ dependent, as the TRPM7i either by 2APB treatment or CRISPR/Cas9-mediated gene knockdown reduced $\mathrm{Ca}^{2+}$ influx (Figs. $2 \mathrm{a}$ and $3 \mathrm{~h}$ ) in concomitant with the decrease in cell motility (Figs. 1-3). Notably, the dominant role of $\mathrm{Ca}^{2+}$ in NSCLC cell motility was observed upon addition of extracellular $\mathrm{Ca}^{2+}$ following ion chelation by EDTA (Supplementary Fig. S16).

Alterations of O-GIcNAc cycling enzymes OGT and OGA, as well as an elevated level of O-GlcNAcylation, have been observed in various solid cancers, including breast, colon, pancreas, liver and lung. ${ }^{18,30-32}$ We previously demonstrated that hyper-O-GIcNAcylation renders NSCLC cells to acquired cisplatin resistance via the regulation of p53 and c-Myc. ${ }^{21}$ We herein extend the knowledge that O-GIcNAcylation also regulates NSCLC cell motility, as shown by a decrease in cell motility upon OGT knockdown, which in turn reduces $\mathrm{O}$-GIcNAcylation (Fig. 3e, f). TRPM7 was further found to affect $O$-GlcNAcylation in part through the modulation of $O$ GlcNAc cycling enzymes (Figs. $3 \mathrm{~b}$ and 4 and Supplementary Fig. S9). Most importantly, hyper-O-GlcNAcylation by thiamet $G$ was able to rescue the reduced migratory phenotype upon TRPM7 inhibition (Fig. 4a, b), thereby uncovering the novel regulation of NSCLC cell motility through TRPM7/O-GlcNAc regulatory axis. In non-cancer-related events, the interplay between calcium signalling and $O$-GlcNAcylation has earlier been reported in glucagonstimulated liver autophagy and metabolic adaptation. ${ }^{46}$ That is, glucagon induces $\mathrm{Ca}^{2+}$ release via an intracellular $\mathrm{Ca}^{2+}$ channel InsP3R1 and activates CaMKII, of which phosphorylates OGT and promotes $\mathrm{O}$-GIcNAcylation and activation of Ulk1, a key regulator in autophagy initiation.

Cav-1 is an essential structural protein component of the plasma membrane microdomains called caveolae, which is shown to be upregulated in lung carcinoma, associating with their invasiveness and poor survival of patients. ${ }^{29,36,47}$ We previously reported that Cav-1 plays an essential role in cancer stem cell regulation and their aggressive phenotypes, including enhanced cell migration and invasion, in an experimental model of lung tumorigenesis. ${ }^{48}$ Here we demonstrated a concomitant decrease in cell motility and Cav-1 expression upon TRPM7 inhibition, which could be reversed by the reactivation of $O$-GlcNAcylation (Figs. 4, 5), defining the TRPM7/O-GlcNAc/Cav-1 pathway. Recently, the crosstalk between $O$-GlcNAcylation and ubiquitination has been identified to cause either increased protein stability or turnover. Hypo-O-GlcNAcylation of Cav-1 following TRPM7 inhibition posttranslationally was shown to promote its ubiquitination and subsequent proteasomal degradation (Fig. 5). To our knowledge, this is the first demonstration of the regulation of Cav-1 by $\mathrm{O}$ GlcNAcylation and ubiquitination.

In lung cancer, a frequent gene amplification or copy number gain of MYC was detected in small cell lung cancer in up to $40 \%$ of all cases and in NSCLC in human tumour tissues and 
a
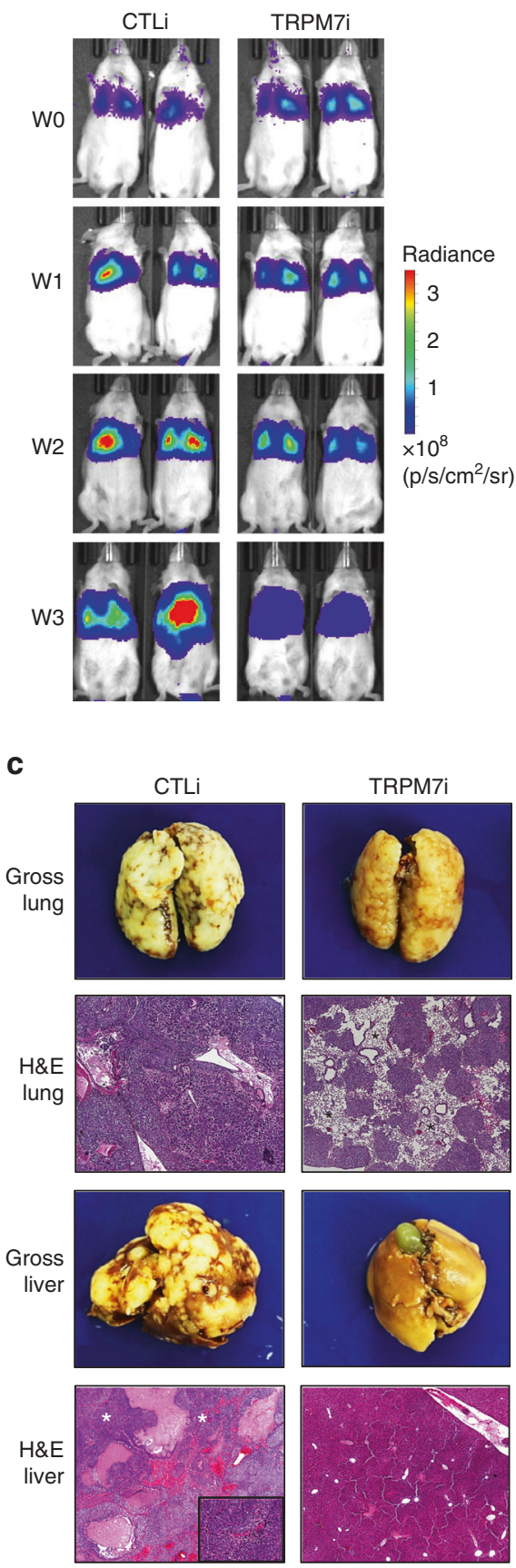

b

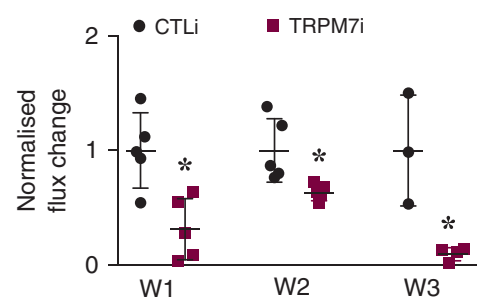

d
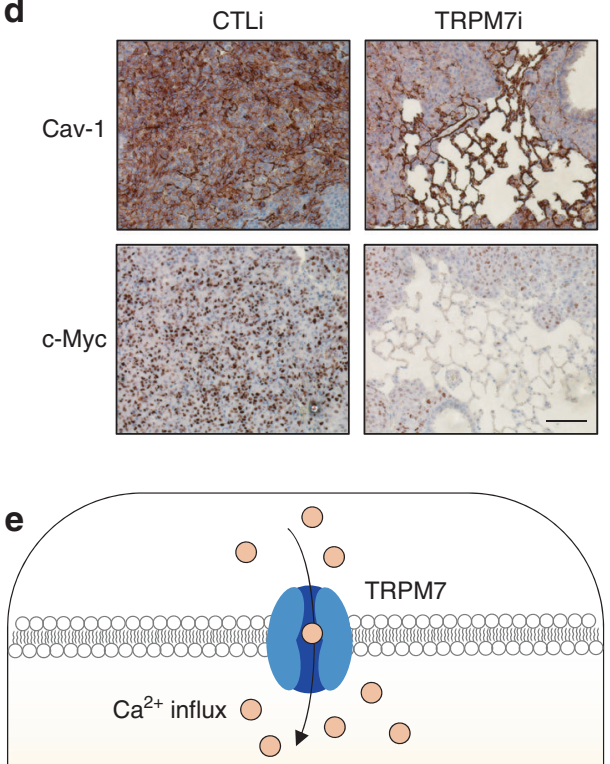

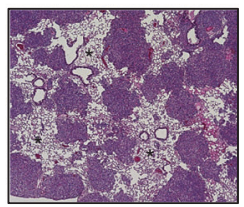

TRPM7i

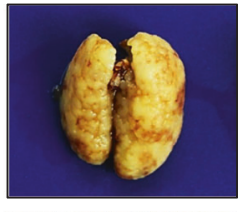

Cav-1
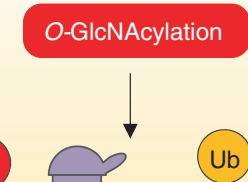

Ub

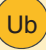

Proteasome

Ub)
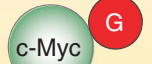

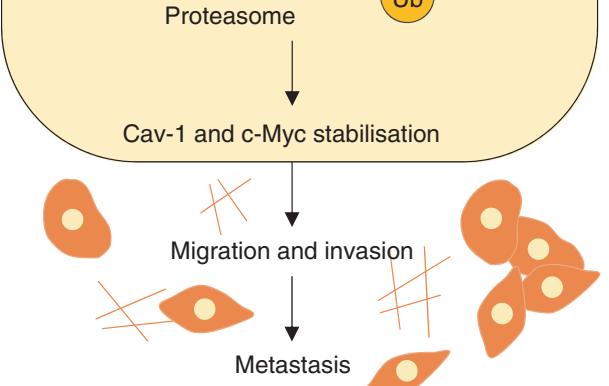

Fig. 6 Inhibition of TRPM7 suppresses experimental lung cancer metastasis in vivo. Luciferase-labelled TRPM7i or CTLi NCI-H292 cells were injected into NSG mice via tail vein. a Representative bioluminescence of mice taken at the time of inoculation (week 0) and at 1,2 and 3 weeks post injection. b Quantitative analysis of bioluminescence signals over time. Plots are fold difference relative to CTLi cells after normalisation to their initial signal at the time of inoculation. Data are mean \pm s.d. $(n=5) .{ }^{*} p<0.05$ versus CLTi cells; two-sided Student's $t$ test. c Representative gross pathology and H\&E micrographs of lung and liver tissues. (Upper) Asterisks show larger alveolar space without metastatic foci. (Lower) Asterisks show hypercellular neoplastic lesions. d IHC analysis of Cav-1 and c-Myc in isolated lungs from mice bearing CTLi and TRPM7i cells. Scale bar $=100 \mu \mathrm{m}$ (see also Supplementary Fig. S15 for the IHC analysis of TRPM7) e A schematic working model for the function of TRPM7/O-GICNAc regulatory axis in NSCLC cell motility and metastasis. TRPM7 regulates $\mathrm{Ca}^{2+}$ influx in NSCLC cells, resulting in an increase of O-GlcNAcylation of Cav-1 and c-Myc and their stabilisation. G O-GlcNAcylation, Ub ubiquitination.

various animal models. ${ }^{49}$ MYC gain is associated with lymph node metastasis and is believed to be a metastasis gene for NSCLC. ${ }^{37}$ Like Cav-1, c-Myc was found in this study to be a favourable target of TRPM7 and O-GlcNAcylation in controlling NSCLC cell motility. A crosstalk between $O-G I c N A c y l a t i o n$ and ubiquitination of c-Myc was also observed (Figs. 4, 5). When O-GlcNAcylation is repressed by TRPM7 inhibition, c-Myc ubiquitination escalates, leading to increased proteasomal degradation. Previous tumorigenesis and metastasis-promoting gene signalling network based on wholetranscriptome gene expression profiles comparing normal lung 
epithelial cells versus transformed lung cells revealed $M Y C$ overexpression and its first-order linkage to CAV1 at the focal position of the network, indicating the potential complex interaction between c-Myc and Cav-1 in the progression of lung carcinoma. ${ }^{48}$ Having demonstrated that TRPM7 acts upstream of the regulatory axis mediating cell motility via c-Myc and Cav-1, we further showed that TRPM7 inhibition could indeed suppress experimental lung metastases (Fig. 6), advising the potential therapeutic application of TRPM7 inhibition for advanced lung cancer.

In summary, the evidence presented here demonstrates that NSCLC cell motility could be modulated by TRPM7 and $\mathrm{Ca}^{2+}$ influx through O-GlcNAcylation. Hyper-O-GlcNAcylation of Cav-1 and cMyc stabilises the proteins by interfering with their ubiquitination process and subsequent proteasomal degradation, as schematically summarised in Fig. 6e. Our novel findings on TRPM7/OGlcNAc regulatory axis provide an integrated understanding of how calcium signalling links to O-GlcNAc signalling and likely dysregulated metabolism, which could aid in the complex understanding of lung cancer progression and metastasis and lay groundwork for future novel therapeutics. Targeting this regulatory axis and downstream targets could be advantageous for the treatment of advanced lung cancer and other related malignancies.

\section{ACKNOWLEDGEMENTS}

S.I. is a Senior Research Scholar of Thailand Research Fund. We would like to acknowledge Profs. Davor Solter and Barbara Knowles for their comments on the manuscript. We thank Drs. Arnatchai Maiuthed and Ornjira Prakhongcheep for their technical assistance.

\section{AUTHOR CONTRIBUTIONS}

S.L. initialised the project, designed research, performed bioinformatics analysis, carried out the major experiments, coordinated the project and prepared the manuscript. N.R. carried out the in vitro experiments. P.S. performed the animal experiments. C.V. derived primary lung cancer cells. S.K. performed IHC staining. P.C. and Y.R. participated in the design of the study. S.I. supervised the project and prepared the manuscript. All authors read and approved the final manuscript.

\section{ADDITIONAL INFORMATION}

Ethics approval and consent to participate This study was approved by the Ethics Committee of the Faculty of Medicine, Chulalongkorn University (IRB 365/62) and performed in accordance with the Declaration of Helsinki. The donors are informed completely with their consent. All cell lines used in this study were purchased from American Type Culture Collection (ATCC, Manassas, VA). All animal experiments were performed in accordance with Guidelines for Animal Experiments at West Virginia University with the approval of the Institutional Animal Care and Use Committee (\#1702005551).

Data availability The datasets used and/or analysed during the current study are available from the corresponding author on reasonable request. Supplementary information is available at the British Journal of Cancer website.

Competing interests The authors declare no competing interests.

Funding information This work was supported by grants from Thailand Research Fund (RSA6280103, to S.L.), the Commission on Higher Education (CHE-RES-RG-49, to S.I.), the Royal Golden Jubilee Ph.D. Programme (PHD/0085/2558, to P.S.) and $\mathrm{NIH}$ (R01-ES022968, to Y.R.; P20-RR016440 and P30-RR032138/GM103488, to WVU Animal Models \& Imaging Facility).

Supplementary information is available for this paper at https://doi.org/10.1038/ s41416-020-0991-7.

Publisher's note Springer Nature remains neutral with regard to jurisdictional claims in published maps and institutional affiliations.

\section{REFERENCES}

1. Berridge, M. J., Bootman, M. D. \& Roderick, H. L. Calcium signalling: dynamics, homeostasis and remodelling. Nat. Rev. Mol. Cell. Biol. 4, 517-529 (2003).

2. Tonelli, F. M., Santos, A. K., Gomes, D. A., da Silva, S. L., Gomes, K. N., Ladeira, L. O. et al. Stem cells and calcium signaling. Adv. Exp. Med. Biol. 740, 891-916 (2012).

3. Zhivotovsky, B. \& Orrenius, S. Calcium and cell death mechanisms: a perspective from the cell death community. Cell Calcium 50, 211-221 (2011).

4. Stewart, T. A., Yapa, K. T. \& Monteith, G. R. Altered calcium signaling in cancer cells. Biochim. Biophys. Acta 1848, 2502-2511 (2015).

5. Maklad, A., Sharma, A. \& Azimi, I. Calcium signaling in brain cancers: roles and therapeutic targeting. Cancers (Basel) 11, 145 (2019).

6. Yang, H., Zhang, Q., He, J. \& Lu, W. Regulation of calcium signaling in lung cancer. J. Thorac. Dis. 2, 52-56 (2010).

7. Blandin Knight, S., Crosbie, P. A., Balata, H., Chudziak, J., Hussell, T. \& Dive, C. Progress and prospects of early detection in lung cancer. Open Biol. 7, 170070 (2017).

8. Friedl, P. \& Wolf, K. Tumour-cell invasion and migration: diversity and escape mechanisms. Nat. Rev. Cancer 3, 362-374 (2003).

9. Palmer, T. D., Ashby, W. J., Lewis, J. D. \& Zijlstra, A. Targeting tumor cell motility to prevent metastasis. Adv. Drug Deliv. Rev. 63, 568-581 (2011).

10. Déliot, N. \& Constantin, B. Plasma membrane calcium channels in cancer: alterations and consequences for cell proliferation and migration. Biochim. Biophys. Acta 1848, 2512-2522 (2015).

11. Hantute-Ghesquier, A., Haustrate, A., Prevarskaya, N. \& Lehen'kyi, V. TRPM family channels in cancer. Pharmaceuticals 11, 58 (2018).

12. Fiorio Pla, A. \& Gkika, D. Emerging role of TRP channels in cell migration: from tumor vascularization to metastasis. Front. Physiol. 4, 311 (2013).

13. Liu, K., Xu, S. H., Chen, Z., Zeng, Q. X., Li, Z. J. \& Chen, Z. M. TRPM7 overexpression enhances the cancer stem cell-like and metastatic phenotypes of lung cancer through modulation of the Hsp90a/uPA/MMP2 signaling pathway. BMC Cancer 18, 1167 (2018)

14. Frischauf, I., Fahrner, M., Jardín, I. \& Romanin, C. The STIM1: Orai interaction. Adv. Exp. Med. Biol. 898, 25-46 (2016).

15. Hanahan, D. \& Weinberg, R. A. Hallmarks of cancer: the next generation. Cell 144, 645-674 (2011).

16. Cheong, H., Lu, C., Lindsten, T. \& Thompson, C. B. Therapeutic targets in cancer cell metabolism and autophagy. Nat. Biotechnol. 30, 671-678 (2012).

17. Hart, G. W. Three decades of research on O-GlcNAcylation-a major nutrient sensor that regulates signaling, transcription and cellular metabolism. Front. Endocrinol. 5, 183 (2014).

18. de Queiroz, R. M., Carvalho, E. \& Dias, W. B. O-GlcNAcylation: the sweet side of the cancer. Front. Oncol. 4, 132 (2014).

19. Győrffy, B., Surowiak, P., Budczies, J. \& Lánczky, A. Online survival analysis software to assess the prognostic value of biomarkers using transcriptomic data in non-small-cell lung cancer. PLOS ONE 8, e82241 (2013).

20. Li, Q., Birkbak, N. J., Gyorffy, B., Szallasi, Z. \& Eklund, A. C. Jetset: selecting the optimal microarray probe set to represent a gene. BMC Bioinform. 12, 474 (2011).

21. Luanpitpong, S., Angsutararux, P., Samart, P., Chanthra, N., Chanvorachote, P. \& Issaragrisil, S. Hyper-O-GIcNAcylation induces cisplatin resistance via regulation of p53 and c-Myc in humanlung carcinoma. Sci. Rep. 7, 10607 (2017).

22. Doench, J. G., Fusi, N., Sullender, M., Hegde, M., Vaimberg, E. W., Donovan, K. F. et al. Optimized sgRNA design to maximize activity and minimize off-target effects of CRISPR-Cas9. Nat. Biotechnol. 34, 184-191 (2016).

23. Sanjana, N. E., Shalem, O. \& Zhang, F. Improved vectors and genome-wide libraries for CRISPR screening. Nat. Methods 11, 783-784 (2014).

24. Stewart, S. A., Dykxhoorn, D. M., Palliser, D., Mizuno, H., Yu, E. Y., An, D. S. et al. Lentivirus-delivered stable gene silencing by RNAi in primary cells. RNA 9, 493-501 (2003).

25. Liu, H., Dilger, J. P. \& Lin, J. The role of transient receptor potential melastatin 7 (TRPM7) in cell viability: A potential target to suppress breast cancer cell cycle. Cancers (Basel) 12, 131 (2020).

26. Chokshi, R., Fruasaha, P. \& Kozak, J. A. 2-Aminoethyl diphenyl borinate (2-APB) inhibits TRPM7 channels through an intracellular acidification mechanism. Channels (Austin) 6, 362-369 (2012).

27. Chubanov, V., Schäfer, S., Ferioli, S. \& Gudermann, T. Natural and synthetic modulators of the TRPM7 channel. Cells 3, 1089-1101 (2014).

28. Lin, C. H., Jackson, A. L., Guo, J., Linsley, P. S. \& Eisenman, R. N. Myc-regulated microRNAs attenuate embryonic stem cell differentiation. EMBO J. 28, 3157-3170 (2009).

29. Luanpitpong, S., Talbott, S. J., Rojanasakul, Y., Nimmannit, U., Pongrakhananon, V., Wang, L. et al. Regulation of lung cancer cell migration and invasion by reactive oxygen species and caveolin-1. J. Biol. Chem. 285, 38832-38840 (2010).

30. Lynch, T. P., Ferrer, C. M., Jackson, S. R., Shahriari, K. S., Vosseller, K. \& Reginato, M. J. Critical role of O-Linked $\beta$-N-acetylglucosamine transferase in prostate cancer invasion, angiogenesis, and metastasis. J. Biol. Chem. 287, 11070-11081 (2012). 
31. Ferrer, C. M., Lu, T. Y., Bacigalupa, Z. A., Katsetos, C. D., Sinclair, D. A. \& Reginato, M. J. O-GlcNAcylation regulates breast cancer metastasis via SIRT1 modulation of FOXM1 pathway. Oncogene 36, 559-569 (2017).

32. Jiang, M., Xu, B., Li, X., Shang, Y., Chu, Y., Wang, W. et al. O-GlcNAcylation promotes colorectal cancer metastasis via the miR-101-O-GlcNAc/EZH2 regulatory feedback circuit. Oncogene 38, 301-316 (2019).

33. Guo, C. B., Wang, S., Deng, C., Zhang, D. L., Wang, F. L. \& Jin, X. Q. Relationship between matrix metalloproteinase 2 and lung cancer progression. Mol. Diagn. Ther. 11, 183-192 (2007).

34. Wang, Y., Xu, X., Song, C., Wu, J., Hu, X., Zhu, H. et al. ATIQCTPC targeting MMP-9: a key step to slowing primary tumor growth and inhibiting metastasis of lewis lung carcinoma in vivo. Oncotarget 8, 63881-63889 (2017).

35. Luanpitpong, S., Li, J., Manke, A., Brundage, K., Ellis, E., McLaughlin, S. L. et al. SLUG is required for SOX9 stabilization and functions to promote cancer stem cells and metastasis in human lung carcinoma. Oncogene 35, 2824-2833 (2016).

36. Ho, C. C., Huang, P. H., Huang, H. Y., Chen, Y. H., Yang, P. C. \& Hsu, S. M. Upregulated caveolin-1 accentuates the metastasis capability of lung adenocarcinoma by inducing filopodia formation. Am. J. Pathol. 161, 1647-1656 (2002).

37. Rapp, U. R., Korn, C., Ceteci, F., Karreman, C., Luetkenhaus, K., Serafin, V. et al. MYC is a metastasis gene for non-small-cell lung cancer. PLOS ONE 4, e6029 (2009).

38. Li, M. D., Ruan, H. B., Hughes, M. E., Lee, J. S., Singh, J. P., Jones, S. P. et al. OGICNAc signaling entrains the circadian clock by inhibiting BMAL1/CLOCK ubiquitination. Cell Metab. 17, 303-310 (2013).

39. Luanpitpong, S., Chanthra, N., Janan, M., Poohadsuan, J., Samart, P., U-Pratya, Y et al. Inhibition of O-GIcNAcase sensitizes apoptosis and reverses bortezomib resistance in mantle cell lymphoma through modification of truncated Bid. Mol. Cancer Ther. 17, 484-496 (2018).

40. Huber, S. M. Oncochannels. Cell Calcium 53, 241-255 (2013).

41. Hanahan, D. \& Weinberg, R. A. The hallmarks of cancer. Cell 100, 57-70 (2000).

42. Buchholz, M., Schatz, A., Wagner, M., Michl, P., Linhart, T., Adler, G. et al. Overexpression of c-myc in pancreatic cancer caused by ectopic activation of NFATc1 and the $\mathrm{Ca}^{2+}$ /calcineurin signaling pathway. EMBO J. 25, 3714-3724 (2006).

43. Sun, Y., Sukumaran, P., Varma, A., Derry, S., Sahmoun, A. E. \& Singh, B. B. Cholesterolinduced activation of TRPM7 regulates cell proliferation, migration, and viability of human prostate cells. Biochim. Biophys. Acta 1843, 1839-1850 (2014).
44. Guilbert, A., Gautier, M., Dhennin-Duthille, I., Rybarczyk, P., Sahni, J., Sevestre, H. et al. Transient receptor potential melastatin 7 is involved in oestrogen receptornegativemetastatic breast cancer cells migration through its kinase domain. Eur. J. Cancer 49, 3694-3707 (2013).

45. Wang, J., Liao, Q. J., Zhang, Y., Zhou, H., Luo, C. H., Tang, J. et al. TRPM7 is required for ovarian cancer cell growth, migration and invasion. Biochem. Biophys. Res. Commun. 454, 547-553 (2014).

46. Ruan, H. B., Ma, Y., Torres, S., Zhang, B., Feriod, C., Heck, R. M. et al. Calciumdependent O-GICNAc signaling drives liver autophagy in adaptation to starvation. Genes Dev. 31, 1655-1665 (2017).

47. Yoo, S. H., Park, Y. S., Kim, H. R., Sung, S. W., Kim, J. H., Shim, Y. S. et al. Expression of caveolin-1 is associated with poor prognosis of patients with squamous cell carcinoma of the lung. Lung Cancer 42, 195-202 (2003).

48. Luanpitpong, S., Wang, L., Stueckle, T. A., Tse, W., Chen, Y. C. \& Rojanasakul, Y. Caveolin-1 regulates lung cancer stem-like cell induction and p53 inactivation in carbon nanotube-driven tumorigenesis. Oncotarget 5, 3541-3554 (2014).

49. Seo, A. N., Yang, J. M., Kim, H., Jheon, S., Kim, K., Lee, C. T. et al. Clinicopathologic and prognostic significance of c-MYC copy number gain in lung adenocarcinomas. Br. J. Cancer 110, 2688-2699 (2014).

(i) Open Access This article is licensed under a Creative Commons Attribution 4.0 International License, which permits use, sharing, adaptation, distribution and reproduction in any medium or format, as long as you give appropriate credit to the original author(s) and the source, provide a link to the Creative Commons license, and indicate if changes were made. The images or other third party material in this article are included in the article's Creative Commons license, unless indicated otherwise in a credit line to the material. If material is not included in the article's Creative Commons license and your intended use is not permitted by statutory regulation or exceeds the permitted use, you will need to obtain permission directly from the copyright holder. To view a copy of this license, visit http://creativecommons. org/licenses/by/4.0/.

(c) The Author(s) 2020 